\title{
Population Structure of Denison's barb, Puntius denisonii (Pisces: Cyprinidae): A Species Complex Endemic to the Western Ghats of India
}

\section{Lijo John ${ }^{1,2 *}$, Reynold Peter ${ }^{2}$ and Gopalakrishnan A}

${ }^{1}$ National Bureau of Fish Genetic Resources (NBFGR), Cochin Unit, CMFRI Campus, Kochi-682018, Kerala, India

${ }^{2}$ Genetics and Genomics Section, Marine Biotechnology Division, Central Marine Fisheries Research Institute, PB No. 1603, Kochi-682018, Kerala, India

\begin{abstract}
Genetic and morphologic variation, haplotype relationships, and structuring of populations within Puntius denisonii and its close related species Puntius chalakkudiensis have been tested using molecular and biometric data, to infer phylogeographic patterns. Sequences of mitochondrial DNA ATPase 8 and 6 genes, and morphometric data, were used to find population structuring. Specimens were collected from 7 locations in the southern region of Western Ghats, a global biodiversity hotspot in India. Biometric analysis revealed apparent heterogeneity in the morphology and color pattern between the species at juvenile and adult stages, and among different geographically separated populations of these species. High values for mean pair wise distances and a high proportion of the total variance attributed to differences between the geographically isolated populations with AMOVA, indicated clear population structuring within these species. Extremely high values for Pair wise $F_{\mathrm{ST}}$ and significantly lower $\mathrm{Nm}$ values observed among the populations studied, suggested little or no effective gene flow among them. Constructed phylogenies further confirmed a high degree of population structuring within the species, showing local endemism with population specific haplotypes forming a species complex. The present study thus estimates the validity of subpopulations within $P$. denisonii and $P$. chalakkudiensis; clarifies the relationships of populations of $P$. denisonii with that of $P$. chalakkudiensis, and also indicates the presence of four different independent evolutionary lineages forming cryptic species within $P$. denisonii. The study further emphasizes the need for a conservation policy to be developed for each population of both species, separately based on MUs (Management Units).
\end{abstract}

Keywords: Puntius denisonii; Western Ghats; Cryptic species; Population structure; Conservation

\section{Introduction}

Puntius denisonii (Pisces: Cyprinidae) is a vibrantly colored, globally traded ornamental teleost, endemic to the southern part of the Western Ghats of India. It is found in selected west flowing rivers originating from the Western Ghats [1-3]. The species was described by Francis Day, in 1865 [4], from Kerala state of India, and was of no interest to commercial fisheries [5], until 1996. In 1997, in Singapore's Aquarama exhibition, the species won an award in the 'new species' category $[3,6]$, and became very popular in both national and international aquarium trade. Later on Menon et al. [1] described another species, Puntius chalakkudiensis, from the River Chalakkudy in the Western Ghats, which closely resembles $P$. denisonii in appearance. Both the species then achieved high demand in domestic, as well as in international aquarium trade, and is being exploited from the wild in large quantities $[7,8]$. Over-fishing, when particularly directed towards certain sizes or age classes, may reduce population sizes to levels at which inbreeding and loss of genetic diversity may become serious problems, or result in extinction of local populations or population segments [9]. Ponniah and Gopalakrishnan [2] reported an alarming rate of depletion of fish diversity of the region due to overexploitation. There is an urgent need for the development of scientific management strategies for the sustainable utilization of these natural resources.

Mitochondrial DNA (mtDNA) sequencing has become the molecular marker of choice, when studying cospecific populations (eg. [10-15]). In recent years, mtDNA, because of its fast evolution [16], has been widely applied in systematics, population genetics, inference of migration routes and conservation biology of animals. In population studies, a hierarchical description of genetic diversity reflects phylogenetic relationships among populations distributed over different geographical regions, or may infer relationships among alleles based on the gene tree of organelle DNA (eg. [16-21]).

Genetic markers are generally oversensitive to a low level of gene flow: a relatively low level of exchange between stocks, negligible from a management perspective, may be sufficient to ensure genetic homogeneity [22-24]. Therefore, molecular markers alone may not be sufficient to detect existing genetic variation among populations, and also only a small proportion of DNA is analyzed by molecular markers. Morphometric and meristic characters have been commonly used in fisheries biology, as powerful phenotypic tool for measuring discreteness and relationships among various taxonomic categories [25-28]. However, phenotypic markers may detect morphological differentiation due to environmental differences in the habitats of partially-isolated stocks, which may be a practical level of partitioning among self-recruiting stocks. Such self-recruiting stocks may react independently to exploitation [24], even without showing genetic differentiation [29]. Morphometric and meristic analysis can thus, be a first step in investigating the stock structure of species with large population sizes [29]. As a potential indicator of phenotypic stocks, analysis of morphometric landmarks is a valuable tool that compliments other stock identification methods. The present study provides a comprehensive phenotypic and genetic analysis of different geographically isolated populations of $P$. denisonii and $P$. chalakkudiensis. The study is a pioneering attempt in determining population/stock structure and diversity of $P$. denisonii, besides genetic comparison of $P$. denisonii with $P$. chalakkudiensis.

*Corresponding author: Lijo John, National Bureau of Fish Genetic Resources (NBFGR), Cochin Unit, CMFRI Campus, Kochi-682018, Kerala, India, E-mail: lijocjohn@yahoo.co.in

Received January 10, 2013; Accepted March 27, 2013; Published April 04, 2013

Citation: John L, Peter R, Gopalakrishnan A (2013) Population Structure of Denison's barb, Puntius denisonii (Pisces: Cyprinidae): A Species Complex Endemic to the Western Ghats of India. J Phylogen Evolution Biol 1: 106. doi:10.4172/2329-9002.1000106

Copyright: (c) 2013 John L, et al. This is an open-access article distributed under the terms of the Creative Commons Attribution License, which permits unrestricted use, distribution, and reproduction in any medium, provided the original author and source are credited. 
Citation: John L, Peter R, Gopalakrishnan A (2013) Population Structure of Denison's barb, Puntius denisonii (Pisces: Cyprinidae): A Species Complex Endemic to the Western Ghats of India. J Phylogen Evolution Biol 1: 106. doi:10.4172/2329-9002.1000106

\section{Materials and Methods}

\section{Sampling}

Fish samples were collected from seven geographically isolated locations, comprising the rivers Chandragiri, Kariangode, Valapattanam, Chaliyar, Chalakkudy, Periyar and Pamba (Figure 1), throughout the species distribution (Table 1). Specimens of $P$. denisonii and $P$. chalakkudiensis were identified up to species level in the field, following Day [4] and Menon et al. [1]. A piece of tissue (fin clips of approximately $5 \times 5 \mathrm{~mm}$ size from pectoral and pelvic fins of right side of the specimens) was excised and placed in $95 \%$ ethanol for isolating DNA, and further the specimens were fixed in $30 \%$ formalin and preserved in $10 \%$ formalin for biometric analysis.

\section{Biometric analysis}

In biometric analysis, color pattern of the juveniles and adult specimens, structure and arrangement of pharyngeal teeth and gill rakers, and morphometric measurements, were thoroughly examined. Except morphometrics, all other characters were examined at species level; whereas, morphometric analysis has been done up to population level. Morphometric measurements following Kottelat [30], were made on 210 specimens comprising 30 each from 7 different watersheds, using 21 reliably measurable morphometric characters described in table 2. Measurements of parts of the head are given as percentages of head length $\left(L_{\mathrm{H}}\right)$. The head length and measurements of other parts of the body are represented as percentages of standard length $\left(L_{\mathrm{S}}\right)$.
The pharyngeal teeth were counted (8 specimens), and represented by a formula adopting the method of Hubbs and Lagler [22]. The structure and arrangement of pharyngeal teeth and gill rakers were observed under a binocular microscope (Nikon DS-L2), with a magnification of $4 \mathrm{X}$, and images were digitally captured.

In morphometric analyses, size-dependent variation for morphometric characters was excluded [31]. Both univariate and multivariate analysis of variance were carried out to test the significance of morphometric differences among populations. The descriptive statistics viz. minimum, maximum, mean and standard deviation for morphometric traits were estimated using SPSS software (ver. 13.0). The Coefficient of Variation (CV) was computed for each character, following van Valen [32]. In each species' sample group, morphological variability was estimated by the multivariate generalization of the coefficient of variation (CVp), using the formula of van Valen [32]. To identify whether there are any statistically significant differences between the species/population for each character, one-way Analysis of Variance (ANOVA) was performed [33], using SPSS software (ver. 13.0). Finally, the size-adjusted morphometric data showing statistically significant differences among groups (species or populations) were submitted to Principal Component Analysis (PCA), and scatter plots generated using the software PAST (ver. 1.89).

\section{Genetic analysis}

DNA isolation and PCR amplification: Total DNA was extracted

\begin{tabular}{|c|c|c|c|c|c|c|c|}
\hline \multirow{2}{*}{$\begin{array}{l}\text { River basin } \\
\text { Chandragiri }\end{array}$} & \multirow{2}{*}{$\begin{array}{l}\text { Site } \\
\text { Sullya }\end{array}$} & \multirow{2}{*}{$\begin{array}{c}\text { Site code } \\
\text { CDR }\end{array}$} & \multicolumn{2}{|c|}{ Geographic coordinates } & \multirow{2}{*}{$\begin{array}{l}n_{t} \\
50\end{array}$} & \multirow{2}{*}{$\begin{array}{c}\text { Mt DNA (ATPase } 8 / 6 \text { ) } \\
10\end{array}$} & \multirow{2}{*}{$\begin{array}{c}\text { Morphometrics } \\
30\end{array}$} \\
\hline & & & $12^{\circ} 34^{\prime} \mathrm{N}$ & $75^{\circ} 23^{\prime} \mathrm{E}$ & & & \\
\hline Kariangode & Cherupuzha & KGD & $12^{\circ} 17^{\prime} \mathrm{N}$ & $75^{\circ} 23^{\prime} \mathrm{E}$ & 32 & 8 & 30 \\
\hline Valapattanam & Koottupuzha & VLP & $12^{\circ} 04^{\prime} \mathrm{N}$ & $75^{\circ} 43^{\prime} \mathrm{E}$ & 52 & 8 & 30 \\
\hline Chaliyar & Pullooranpara & CLR & $11^{\circ} 23^{\prime} \mathrm{N}$ & $76^{\circ} 01^{\prime} \mathrm{E}$ & 36 & 9 & 30 \\
\hline Chalakkudy & Athirapilly & $\mathrm{CHD}$ & $10^{\circ} 17^{\prime} \mathrm{N}$ & $76^{\circ} 32^{\prime} \mathrm{E}$ & 35 & 9 & 30 \\
\hline Periyar & Pooyamkutty & PER & $10^{\circ} 08^{\prime} \mathrm{N}$ & $76^{\circ} 47^{\prime} \mathrm{E}$ & 55 & 17 & 30 \\
\hline Pamba & Kuzhimavu & PMB & $09^{\circ} 27^{\prime} \mathrm{N}$ & $76^{\circ} 57^{\prime} \mathrm{E}$ & 32 & 13 & 30 \\
\hline
\end{tabular}

$n_{t}$-Total no. of specimens (including juveniles) collected from each location.

Table 1: Details of sampling with geographic locations and numbers of individuals used in analyses.

\begin{tabular}{|c|c|c|}
\hline Character & Code & Description \\
\hline Standard length & $L_{s}$ & snout tip to the midpoint of caudal fin origin \\
\hline Head length & $L_{H}$ & snout tip to the posterior edge of operculum \\
\hline Maximum body depth & MBD & distance between points at deepest part of body (measured vertically) \\
\hline Pre dorsal length & $L_{\text {PRD }}$ & snout tip to the origin of dorsal fin \\
\hline Post dorsal length & $L_{\mathrm{PD}}$ & length from the last ray of the dorsal fin to origin of caudal fin \\
\hline Pre ventral length & $L_{\mathrm{PRV}}$ & snout tip to the origin of ventral fin \\
\hline Pre anal length & $L_{\text {PRA }}$ & snout tip to the origin of anal fin \\
\hline Pectoral to pelvic origin & $L_{\text {PTFPLF }}$ & length from the origin of pectoral fin to that of pelvic fin \\
\hline Pelvic to anal & $L_{\text {PLFAF }}$ & length from the origin of pelvic fin to that of anal fin \\
\hline Length of body cavity & $L_{\mathrm{BC}}$ & length from the first ray of pectoral fin to vent \\
\hline Dorsal fin base & FBDO & length between the visible origins of the first spine and the last ray of the dorsal fin \\
\hline Anal fin base & FBAN & length between the visible origins of the first spine and the last ray of the anal fin \\
\hline Length of caudal peduncle & $L_{C P}$ & length from the anal fin insert to the midpoint of the caudal peduncle \\
\hline Depth of caudal peduncle & CPD & least depth measured vertically \\
\hline Head depth & HD & depth at the nape measured vertically \\
\hline Width of head & $\mathrm{HW}$ & Width behind orbit perpendicular to the longitudinal axis \\
\hline Width of mouth & MW & length between the points of lateral edges of jaws of the mouth \\
\hline Orbital length & $L_{0}$ & length (along axis) of the orbit \\
\hline Pre orbital length & $L_{\text {PRO }}$ & mouth tip to anterior edge of orbit \\
\hline Post orbital length & $L_{\mathrm{PO}}$ & posterior edge of orbit to posterior edge of operculum \\
\hline Barbel length & $L_{\mathrm{MB}}$ & length of maxillary barbel on left side \\
\hline
\end{tabular}

Table 2: Description of morphometric characteristics studied. 
Citation: John L, Peter R, Gopalakrishnan A (2013) Population Structure of Denison's barb, Puntius denisonii (Pisces: Cyprinidae): A Species Complex Endemic to the Western Ghats of India. J Phylogen Evolution Biol 1: 106. doi:10.4172/2329-9002.1000106

from the tissue (fin clips) samples preserved in 95\% ethanol, following the salting out procedure of Miller et al. [34], after removing ethanol by air drying and washing in Tris buffer ( $\mathrm{pH} 8.0)$. The entire (approx. 950 bp) ATP synthase 8 (ATPase 8) and ATP synthase 6 (ATPase 6) genes were PCR amplified, using primers of Page et al. [35]. Amplifications were performed in $50 \mu \mathrm{l}$ total reaction volume containing $5 \mu \mathrm{l} 10 \mathrm{X}$ PCR buffer (SIGMA-ALDRICH, USA), with $15 \mathrm{mM} \mathrm{MgCl}, 2 \mu$ each of $10 \mu \mathrm{M}$ forward and reverse primers, $1 \mu \mathrm{l} 10 \mathrm{mM}$ dNTPs, $1 \mu \mathrm{l}(3 \mathrm{U})$ Taq DNA polymerase (SIGMA-ALDRICH, USA) and $2 \mu \mathrm{l}(\sim 40 \mathrm{ng})$ of template DNA. The thermal cycling conditions were as follows: an initial denaturation at $95^{\circ} \mathrm{C}$ for $3 \mathrm{~min}$, denaturation at $95^{\circ} \mathrm{C}$ for $30 \mathrm{~s}$, annealing at $56^{\circ} \mathrm{C}$ for $30 \mathrm{~s}$, extension at $72^{\circ} \mathrm{C}$ for $1 \mathrm{~min}$, repeated for 35 cycles, followed by a final extension for $7 \mathrm{~min}$ at $72^{\circ} \mathrm{C}$. Following amplification, $2 \mu \mathrm{l}$ of the PCR products were visualized on $1.5 \%$ agarose gel.

Sequencing and sequence analysis: All PCR products were purified using GenElute PCR Clean-Up Kit (SIGMA), and directly sequenced using the same forward and reverse primers using $\mathrm{ABI}$ Prism Big Dye Terminator v3.1 Cycle Sequencing kit, on an AB 3730 XL capillary sequencer (Applied Biosystems), following manufacturer's instructions. Sequences were edited and the contigs were assembled using BioEdit sequence alignment editor version 7.0.5.2 [36]. Multiple alignments of sequences were performed using ClustalW facility, available in the BioEdit program. Alignment was then manually checked and corrected. Nucleotide sequence characteristics after alignment were analyzed using the program DnaSP version 5.10 [37].

Population genetic analysis: Intra-population diversity was analyzed by estimating gene diversity $(h)$ [37], and nucleotide diversity $(\pi)[38,39]$. Hierarchical genetic differentiation and the significance of group and population structure were tested using Analysis of Molecular Variance (AMOVA) [40], and F-statistics [41], respectively. Samples collected from the same site were treated as a single population sample, except for Periyar (PER). Specimens from Periyar consisted of two morphotypes (Figure 2); one with a black blotch on dorsal fin resembling $P$. chalakkudiensis $[\operatorname{PER}(\mathrm{c})]$, and the other without any black blotch on dorsal fin [PER(d)]. Based on the observations of initial analysis, these morphotypes were considered as different populations/groups in further analysis. This analysis was performed for three hierarchical groupings of the data. The first level compared the variation among individuals within each population. The second level examined genetic structure among populations of each group/ species. The typical P. chalakkudiensis (CHD with a black blotch on dorsal fin, from Chalakkudy River) samples and its look alike samples from Periyar $[\mathrm{PER}(\mathrm{c})]$ and Pamba $[\mathrm{PMB}]$, were combined in Group A $(P c)$. All other samples of $P$. denisonii [CDR, KGD, VLP, CLR and $\operatorname{PER}(\mathrm{d})]$, were combined in Group B $(P d)$. Finally, variation among groups/species, $P$. denisonii and $P$. chalakkudiensis was determined by combining all geographical samples. This analysis provided insight into the proportion of genetic variation attributable to within-population $\left(\Phi_{\mathrm{ST}}\right)$, within-group $\left(\Phi_{\mathrm{SC}}\right)$, and among-group $\left(\Phi_{\mathrm{CT}}\right)$ differences. Pairwise $F_{\mathrm{ST}}$-values and migration rates/gene flow $(\mathrm{Nm})$ were also calculated among the different populations. All population analyses were performed using Arlequin version 3.0 [42].

Phylogenetic analysis: Phylogenetic and molecular evolutionary analyses were conducted using MEGA 5 [43]. Sequence data was subsequently analyzed using Maximum Likelihood, Maximum Parsimony and Neighbour-Joining methods, with Puntius conchonius and Puntius ticto as outgroups. The robustness of the internal nodes of phylogenetic trees was verified using bootstraps of 1000 replicates. Pairwise sequence divergence among populations was calculated according to Kimura 2 parameter (K2P) model [44].

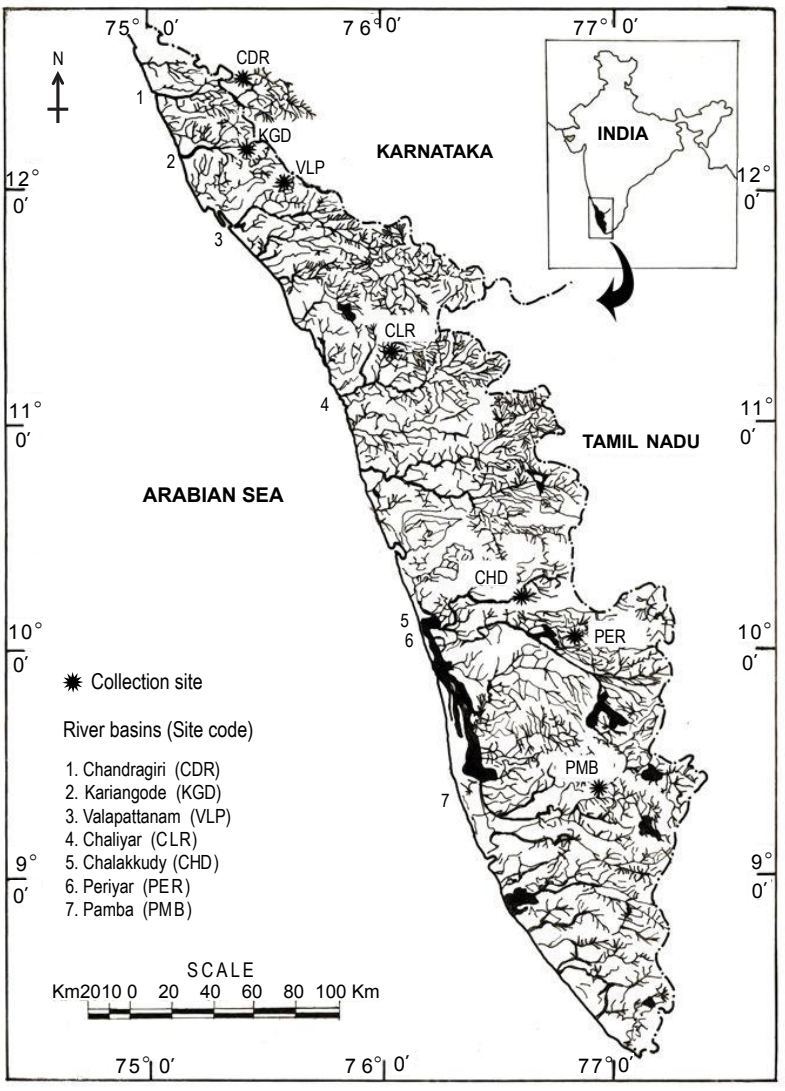

Figure 1: Map of Kerala (reproduced from the Water Atlas of Kerala), showing the distribution of the sampling sites.

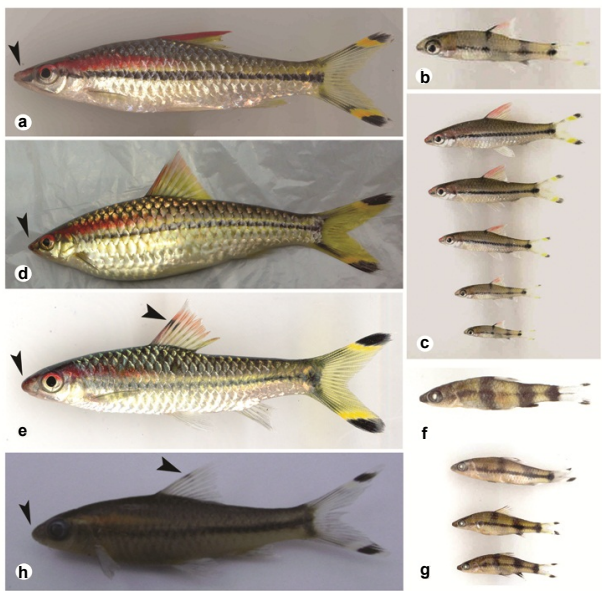

Figure 2: Adult and juvenile specimens collected from different sites; a-Specimen from Valapattanam River (VLP) (total length $102 \mathrm{~mm}$ ); b-Juvenile specimen from Valapattanam River (VLP) (total length $20 \mathrm{~mm}$ ); c-Juvenile specimens from Valapattanam River (VLP) (total length ranges $20-40 \mathrm{~mm}$ ) d-Specimen from Periyar River (PER), without a black blotch on dorsal fin (total length $150 \mathrm{~mm}$ ); e-Typical $P$. chalakkudiensis specimen from Chalakkudy River (CHD) (total length $90 \mathrm{~mm}$ ); f-Typical juvenile specimen of $P$. chalakkudiensis from Chalakkudy River (CHD) (total length $25 \mathrm{~mm}$, formalin preserved); g-Juvenile specimens of $P$. chalakkudiensis from Periyar River (PER) (total length ranges 20-30 mm, formalin preserved); $h$-Specimen from Pamba River (PMB), with a typical black blotch on dorsal fin (total length 85 $\mathrm{mm}$, formalin preserved) 


\section{Results}

\section{Basic ecological observations}

Difference in size, body shape and color among the samples obtained from different sampling sites were readily noticeable (Figure 2). Especially the specimens from North Kerala and Karnataka (NK) region of the Western Ghats, which includes CDR, KGD, VLP and CLR population samples, were easily distinguishable from that of Central and South Kerala (SK) region specimens (includes CHD, PER and PMB population samples). Body of typical $P$. chalakkudiensis specimens was moderately deep (especially in larger specimens), with slightly rounded snout and comparatively more greenish dorsum than NK specimens. A black blotch on the dorsal fin was also prominent in these specimens. But, in $P$. denisonii specimens from North Kerala and Karnataka regions, body was comparatively slender and streamlined with a pointed snout. The body length of $P$. denisonii was relatively shorter (max. total length observed $135 \mathrm{~mm}$ ) than that of $P$. chalakkudiensis (max. total length observed $176 \mathrm{~mm}$ ), as observed in the present study. In addition, P. chalakkudiensis (Figure 2e) has a black longitudinal stripe from the snout, extending along the lateral line, more clearly defined from post orbit to the caudal peduncle, while a clearly defined black longitudinal stripe starts from the side of the snout, passing through mid orbit and abruptly, ending at caudal peduncle is distinct in $P$. denisonii (Figure 2a). Some specimens collected from Periyar River and the specimens from Pamba resembled $P$. chalakkudiensis in their color characteristics (Figure 2). Periyar River is found to be a typical habitat from where two types of specimens obtained; one of which resembled the typical $P$. chalakkudiensis in their morphology, and the second type (Figure 2c) showed more resemblance to $P$. denisonii, without a black blotch on dorsal fin.

Juveniles of both species exhibited different color patterns from that observed in adult specimens (Figure $2 \mathrm{~b}, \mathrm{~d}, \mathrm{f}, \mathrm{g}$ and $\mathrm{h}$ ). Shape and color patterns of early juveniles of $P$. denisonii and $P$. chalakkudiensis were also distinguishable between each other. The prominent red or scarlet horizontal band observed in adult specimens was not present in early juveniles, whereas three prominent black vertical bands were observed. The early juveniles of $P$. denisonii (Figure $2 \mathrm{~b}$ and $\mathrm{d}$ ) from NK region possesses comparatively narrow and diffuse vertical bands on the sides of the body, whereas that of $P$. chalakkudiensis (Figure $2 \mathrm{f}$ and h) possess broader and more prominent bands. However, this pattern disappears in sub-adults and adults in both species. The identity of juveniles was confirmed by sequencing ATPase 8 and 6 genes from one representative sample, each from both species, and by comparing it with that of the adult specimens.

\section{Structure and arrangement of pharyngeal teeth and gill rakers}

Structure and arrangement of pharyngeal teeth and gill rakers were examined to find out any variation between the two species viz. P. denisonii (from Valapattanam) and P. chalakkudiensis (from Chalakkudy). Microscopic examination of pharyngeal teeth revealed the presence of an additional fifth pair of teeth in the inner most rows on pharyngeal bones, in addition to the formula given by Day [4], for $P$. denisonii. The pharyngeal teeth was found to be arranged as 5,3,2$2,3,5$ in both species, as observed in the present study. The pharyngeal bones of both left and right sides possess three rows, with two teeth on the outer row, three on the middle and five on the inner. Out of the 5 teeth in the innermost row, four were of similar size, and the fifth was comparatively smaller (Figure 3a). The gill rakers on the first gill arch in $P$. denisonii were slender and villiform in structure (Figure 3c), whereas in $P$. chalakkudiensis, they were comparatively stout with blunt tips (Figure $3 \mathrm{~b}$ ). The second type of specimens from Periyar (PER[d]),

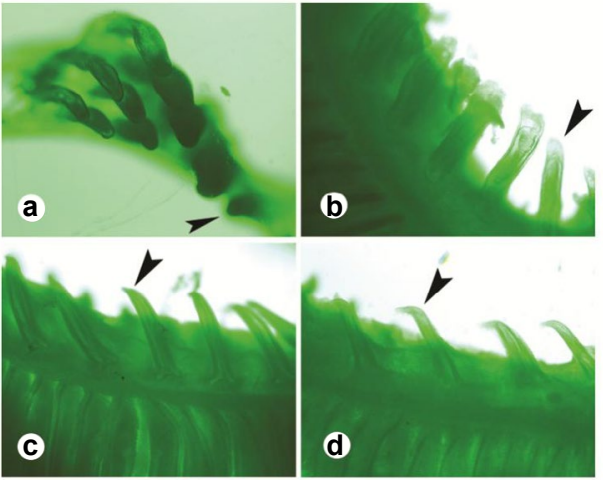

Figure 3: Microscopic images (4X magnification) of pharyngeal teeth and gill rakers of outer gill arch; a-Pharyngeal teeth arranged on one side of the pharyngeal bone of $P$. denisonii specimen from Valapattanam river (arrow shows the smallest fifth teeth); b-Gill rakers of outer gill arch of typical $P$. chalakkudiensis specimen from Chalakkudy river; c-Gill rakers of outer gill arch of $P$. denisonii specimen from Valapattanam river; d-Microscopic image of gill rakers of outer gill arch of specimen without a black blotch on dorsal fin from Periyar river (arrow head on 'b' shows the blunt tip and 'c'and ' $d$ ' shows slender and villiform structure of gill rakers)

without a black blotch on dorsal fin possesses gill rakers, similar to that observed from NK specimens (Figure 3d).

\section{Morphometric traits}

The Coefficient of Variation (CV) for all morphometric traits was generally lower within each population of $P$. denisonii (1.01-9.49\%; Table 3 ) and $P$. chalakkudiensis (1.46-8.75\%; Table 4). The multivariate generalized coefficient of variation $(\mathrm{CVp})$ in each population was also relatively low. Specimens from Periyar showed the highest CVp (5.51\%) among all the populations, even with a relatively low value; indicating minimal or very low intra-population variation. Univariate analysis of variance carried out among populations showed that fish samples from different sites differed significantly (at $p<0.05$ and $p<0.01$ levels of significance), in 18 and 19 out of the 20 morphometric characters examined in P. denisonii (Table 3 ) and P. chalakkudiensis (Table 4), respectively. This indicated heterogeneity in fish morphology among riverine populations of both the species. There was no significant differences $(P>0.05)$ observed in FBDO and $L_{O}$, and MBD among populations of $P$. denisonii and $P$. chalakkudiensis, respectively.

Specimens of $P$. chalakkudiensis collected from its type locality (Chalakkudy river; CHD), and the specimens which resembled the species collected from Periyar (PER) and Pamba (PMB) river systems, were grouped together and compared with other population samples of P. denisonii from NK region (CDR, KGD, VLP and CLR). The univariate Analysis of Variance (ANOVA) showed significant differences at the $\mathrm{p}<0.05$ and $\mathrm{p}<0.01$ levels of significance, in 18 out of 20 morphometric characters (excluding standard length) studied (Table 5). P. denisonii samples from four different sites of NK region shared several of the morphometric characters that are significantly different from those in $P$. chalakkudiensis, with high $\mathrm{F}$ values. For example, the specimens from $\mathrm{NK}$ region have shorter $\mathrm{HD}, \mathrm{MBD}, \mathrm{FBAN}, \mathrm{CPD}$ and $L_{\mathrm{PD}}$. Moreover, larger mean $L_{\mathrm{PRV}}, L_{\mathrm{PRA}}, L_{\mathrm{PTFPLF}}, \mathrm{MW}, L_{\mathrm{PO}}, L_{\mathrm{MB}}$ and $L_{\mathrm{H}}$ were identified in $P$. denisonii specimens from this region; whereas, $P$. chalakkudiensis specimens could be differentiated by larger mean $L_{\mathrm{CP}}, \mathrm{CPD}, \mathrm{HW}, L_{\mathrm{O}}$ and $L_{\mathrm{PRO}}$ (Table 5).

Principal Component Analysis (PCA) was carried out factoring the correlation matrix of the morphometric data between the two 
Citation: John L, Peter R, Gopalakrishnan A (2013) Population Structure of Denison's barb, Puntius denisonii (Pisces: Cyprinidae): A Species Complex Endemic to the Western Ghats of India. J Phylogen Evolution Biol 1: 106. doi:10.4172/2329-9002.1000106

Page 5 of 12

\begin{tabular}{|c|c|c|c|c|c|c|c|c|c|}
\hline & \multicolumn{2}{|l|}{ Chandragiri $(n=30)$} & \multicolumn{2}{|l|}{ Kariangode $(n=30)$} & \multicolumn{2}{|l|}{ Valapattanam $(n=30)$} & \multicolumn{2}{|l|}{ Chaliyar $(n=30)$} & \multirow[b]{2}{*}{$F$ value } \\
\hline & Mean \pm SD (min.-max.) & cV & Mean $\pm \mathrm{SD}$ (min.-max.) & cV & Mean $\pm \mathrm{SD}$ (min.-max.) & cV & Mean $\pm S D$ (min.-max.) & cV & \\
\hline$L_{\mathrm{s}}$ & $74.08 \pm 4.58(67.34-85.70)$ & - & $85.47 \pm 7.2^{\circ}(70.64-98.52)$ & & $.11 \pm 8.18(70.66-101.30)$ & W & $77.67 \pm 5.63(64.55-85.20)$ & 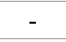 & \\
\hline$L_{H}$ & $24.68^{\mathrm{a}} \pm 0.48(23.85-25.93)$ & 1.94 & $24.26^{\mathrm{ab}} \pm 0.91(22.80-25.63)$ & 3.75 & $3.85^{b} \pm 0.76(22.16-25.11)$ & 3.21 & $23.06^{\circ} \pm 0.66(22.01-24.06)$ & 2.86 & $27.74^{\star *}$ \\
\hline MBD & $23.86^{\mathrm{a}} \pm 0.75(22.82-25.13)$ & 3.14 & $23.93^{\mathrm{ab}} \pm 1.28(21.96-25.71)$ & 5.36 & $24.69^{\mathrm{bc}} \pm 1.33(22.32-26.22)$ & 5.37 & $25.21^{\circ} \pm 0.99(23.42-26.41)$ & 3.93 & $10.06^{\star *}$ \\
\hline$L_{\mathrm{PRD}}$ & $47.64^{\mathrm{a}} \pm 0.62(46.49-49.51)$ & 1.30 & $46.39^{b} \pm 0.81(45.21-47.64)$ & 1.74 & $46.35^{b} \pm 0.69(44.96-47.53)$ & 1.50 & $48.11^{\mathrm{a}} \pm 0.63(47.07-49.37)$ & 1.31 & $49.51^{* *}$ \\
\hline$L_{P D}$ & $37.32^{\mathrm{a}} \pm 1.14(35.49-39.96)$ & 3.05 & $36.88^{\mathrm{ab}} \pm 1.02(35.51-38.74)$ & 2.78 & $36.97^{\mathrm{a}} \pm 1.17(35.15-39.06)$ & 3.18 & $37.72^{\mathrm{ac}} \pm 0.83(36.10-38.90)$ & 2.20 & $3.95^{*}$ \\
\hline$L_{\text {PRV }}$ & $52.52^{\mathrm{a}} \pm 1.12(50.59-54.33)$ & 2.13 & $54.06^{b} \pm 1.62(51.60-56.45)$ & 2.99 & $52.09^{a} \pm 3.18(49.19-58.95)$ & 6.11 & $53.29^{\mathrm{ab}} \pm 0.54(51.12-53.99)$ & 1.01 & $6.31^{\star \star}$ \\
\hline$L_{\text {PRA }}$ & $75.75^{\mathrm{a}} \pm 1.34(72.86-77.51)$ & 77 & $75.14^{\mathrm{ab}} \pm 1.52(72.45-77.49)$ & 2.03 & $74.22^{\mathrm{b}} \pm 1.54(72.31-77.55)$ & 2.08 & $77.11^{\circ} \pm 0.8^{\circ}(75.67-78.88)$ & 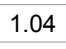 & $4.71^{* *}$ \\
\hline$L_{\text {PTFPLF }}$ & $27.73^{\mathrm{a}} \pm 1.16(25.82-29.51)$ & 4.18 & $27.82^{\mathrm{a}} \pm 1.01(26.02-29.38)$ & 3.62 & $27.20^{\mathrm{a}} \pm 1.16(25.13-29.49)$ & 4.25 & $29.63^{b} \pm 0.81(28.60-30.77)$ & 2.73 & $31.04^{* *}$ \\
\hline$L_{\text {PLFAF }}$ & $23.80^{\mathrm{a}} \pm 0.87(21.89-25.04)$ & 3.66 & $24.71^{\mathrm{bc}} \pm 1.15(22.93-26.50)$ & 4.66 & $24.52^{\mathrm{ab}} \pm 1.25(23.24-26.62)$ & 5.09 & $25.34^{\mathrm{bc}} \pm 0.68(23.46-26.56)$ & 2.68 & $11.79^{* *}$ \\
\hline$L_{\mathrm{BC}}$ & $49.39^{\mathrm{a}} \pm 2.25(45.56-53.25)$ & 4.56 & $48.17^{\mathrm{ab}} \pm 2.09(44.72-51.31)$ & 4.34 & $47.02^{\mathrm{b}} \pm 1.37(45.51-50.62)$ & 2.91 & $51.35^{\circ} \pm 0.78(49.96-52.59)$ & 1.52 & $34.59^{* *}$ \\
\hline FBDO & $16.35^{a} \pm 0.39(15.76-17.01)$ & 2.39 & $16.47^{a} \pm 0.57(15.63-17.21)$ & 3.47 & $16.45^{\mathrm{a}} \pm 0.55$ & 3.32 & $16.36^{\mathrm{a}} \pm 0$ & 5.62 & $0.26 \mathrm{NS}$ \\
\hline FBAN & $08.43^{a} \pm 0.21(08.09-08.88)$ & 2.49 & $08.05^{b} \pm 0.39(07.25-08.74)$ & 4.87 & $08.20^{\mathrm{ab}} \pm 0.46(07.12-08.76)$ & 5.55 & $07.45^{\circ} \pm 0.37(06.83-08.02)$ & 4.97 & $38.13^{* *}$ \\
\hline$L_{C P}$ & $14.31^{\mathrm{a}} \pm 0.73(13.13-15.65)$ & 5.10 & $16.27^{\mathrm{bd}} \pm 1.03(14.61-17.92)$ & 6.32 & $17.38^{\circ} \pm 0.95(15.35-18.67)$ & 5.47 & $15.85^{d} \pm 0.94(14.42-17.39)$ & 5. & $57.30^{* *}$ \\
\hline CPD & $10.74^{\mathrm{a}} \pm 0.35(10.31-11.67)$ & 26 & $11.09^{\mathrm{b}} \pm 0.51(10.41-11.98)$ & 4.59 & $11.33^{c} \pm 0.62(10.48-12.65)$ & 5.49 & $10.82^{\mathrm{ab}} \pm 0.26$ & 2.40 & $10.76^{\star *}$ \\
\hline HD & $60.70^{\mathrm{a}} \pm 1.7^{\circ}(55.55-63.44)$ & 2.80 & $59.70^{\mathrm{a}} \pm 1.85(57.01-62.84)$ & 3.10 & $60.24^{\mathrm{a}} \pm 1.94(56.71-62.92)$ & 3.22 & $64.61^{b} \pm 2.3^{\circ}(61.35-67.85)$ & 3.56 & $38.93^{* *}$ \\
\hline HW & $57.51^{\mathrm{a}} \pm 1.54(52.98-59.96)$ & 2.68 & $54.11^{\mathrm{b}} \pm 1.73(50.77-56.70)$ & 3.21 & $54.24^{\mathrm{b}} \pm 1.47(52.43-56.51)$ & 2.71 & $57.33^{\mathrm{a}} \pm 2.5^{\circ}(54.07-61.95)$ & 4.36 & $30.66^{* *}$ \\
\hline MW & $27.85^{\mathrm{a}} \pm 1.47(24.71-31.23)$ & 5.28 & $24.81^{b} \pm 0.79(23.44-26.15)$ & 3.18 & $24.68^{b} \pm 0.83(22.60-25.84)$ & 3.36 & $27.53^{\mathrm{a}} \pm 2.01(23.05-29.80)$ & 7.30 & $46.64^{\star *}$ \\
\hline$L_{0}$ & $31.32^{\mathrm{a}} \pm 1.72(28.56-33.99)$ & 5.49 & $32.09^{\mathrm{a}} \pm 1.84(28.71-34.22)$ & 5.75 & $31.17^{\mathrm{a}} \pm 1.19(28.83-33.43)$ & 3.83 & $31.21^{\mathrm{a}} \pm 1.48(28.74-32.92)$ & 4.74 & $2.28 \mathrm{NS}$ \\
\hline$L_{\text {PRO }}$ & $32.73^{\mathrm{a}} \pm 1.1^{\circ}(29.80-34.02)$ & 3.36 & $32.28^{\text {ba }} \pm 1.64(29.29-34.50)$ & 5.08 & $31.58^{\mathrm{b}} \pm 1.85(28.97-34.53)$ & 5.86 & $33.75^{\mathrm{ca}} \pm 1.23(31.88-35.88)$ & 3.64 & $11.31^{* *}$ \\
\hline$L_{\mathrm{po}}$ & $38.29^{\mathrm{a}} \pm 1.14(34.98-39.82)$ & 2.98 & $39.53^{b} \pm 1.21(37.53-41.46)$ & 3.07 & $39.64^{b} \pm 1.03(38.15-41.94)$ & 2.61 & $36.10^{\circ} \pm 1.16(34.28-38.53)$ & 3.21 & $62.50^{* *}$ \\
\hline$L_{\text {MB }}$ & $28.55^{\mathrm{a}} \pm 2.07(22.56-30.56)$ & 7.25 & $32.32^{\mathrm{bc}} \pm 3.07(27.92-36.84)$ & 9.49 & $32.87^{\mathrm{cd}} \pm 2.94(28.84-36.99)$ & 8.94 & $34.48^{\mathrm{d}} \pm 2.58(27.73-38.31)$ & 7.48 & $26.10^{\star *}$ \\
\hline$c V p$ & & 4.80 & & 5.32 & & 5.38 & & 4.81 & \\
\hline
\end{tabular}

For each morphometric variable, means with the same letter superscript are not significantly different. See table 2 for explanations of acronyms. * Significant at the $5 \%$ level; ** Significant at the $1 \%$ level; NS, not significant at the $5 \%$ level; SD is standard deviation.

Table 3: Descriptive statistics of transformed morphometric variables, the Coefficient of Variation (CV) of each measurement, the multivariate coefficient of variation of each species $(C V p)$, and F-values (derived from the analysis of variance) of four populations of $P$. denisonii from North Kerala and Karnataka region of Western Ghats.

\begin{tabular}{|c|c|c|c|c|c|c|c|}
\hline & Chalakkudy $(n=30)$ & & Periyar $(n=30)$ & & Pamba $(n=30)$ & & \\
\hline & Mean \pm SD (min. - max.) & cV & Mean \pm SD (min. - max.) & CV & Mean \pm SD (min. - max.) & CV & $F$ value \\
\hline$L_{\mathrm{s}}$ & $100.73 \pm 7.94(85.32-112.44)$ & - & $78.31 \pm 19.69(64.75-129.75)$ & - & $69.16 \pm 12.58(53.60-98.97)$ & - & - \\
\hline$L_{H}$ & $22.21^{\mathrm{a}} \pm 1.27(20.30-24.47)$ & 5.72 & $23.30^{\mathrm{b}} \pm 1.56(19.99-24.88)$ & 6.70 & $23.96^{\mathrm{b}} \pm 0.67(22.55-24.94)$ & 2.80 & $15.50^{\star \star}$ \\
\hline MBD & $26.92^{\mathrm{a}} \pm 0.91(25.48-28.63)$ & 3.40 & $26.98^{\mathrm{a}} \pm 0.81(25.87-28.86)$ & 3.00 & $26.59^{\mathrm{a}} \pm 1.22(24.93-29.30)$ & 4.59 & $1.32 \mathrm{NS}$ \\
\hline$L_{\text {PRD }}$ & $46.11^{\mathrm{a}} \pm 1.33(44.51-48.45)$ & 2.88 & $46.57^{a} \pm 0.97(44.93-48.55)$ & 2.08 & $48.06^{b} \pm 1.07(45.94-50.81)$ & 2.23 & $24.26^{* *}$ \\
\hline$L_{\mathrm{PD}}$ & $41.48^{\mathrm{a}} \pm 1.19(39.66-43.30)$ & 2.86 & $39.49^{b} \pm 1.78(37.51-43.96)$ & 4.51 & $37.76^{c} \pm 1.06(36.48-39.70)$ & 2.81 & $54.59^{\star \star}$ \\
\hline$L_{\mathrm{PRV}}$ & $48.89^{\mathrm{a}} \pm 1.07(47.01-50.52)$ & 2.20 & $50.61^{\mathrm{b}} \pm 1.1^{\circ}(47.67-52.26)$ & 2.17 & $51.92^{\mathrm{c}} \pm 1.08(49.88-53.56)$ & 2.09 & $58.81^{* *}$ \\
\hline$L_{\text {PRA }}$ & $73.04^{\mathrm{a}} \pm 1.58(70.17-75.48)$ & 2.16 & $74.75^{b} \pm 1.39(72.41-77.25)$ & 1.87 & $74.47^{b} \pm 1.08(71.23-75.93)$ & 1.46 & $13.49^{* *}$ \\
\hline$L_{\text {PTFPLF }}$ & $26.11^{\mathrm{a}} \pm 1.57(24.12-28.71)$ & 6.00 & $27.31^{\mathrm{b}} \pm 1.2^{\circ}(25.96-31.03)$ & 4.38 & $27.32^{\mathrm{b}} \pm 0.98(25.32-29.89)$ & 3.59 & $9.04^{* *}$ \\
\hline$L_{\text {PLFAF }}$ & $24.77^{\mathrm{ab}} \pm 0.82(23.32-25.87)$ & 3.31 & $25.33^{\mathrm{a}} \pm 1.28(22.80-29.00)$ & 5.06 & $24.23^{b} \pm 0.87(22.83-25.89)$ & 3.58 & $8.92^{* *}$ \\
\hline$L_{\mathrm{BC}}$ & $49.14^{\mathrm{a}} \pm 2.18(45.43-53.10)$ & 4.44 & $48.60^{\mathrm{a}} \pm 1.84(44.72-53.23)$ & 3.80 & $47.11^{b} \pm 1.36(44.53-50.68)$ & 2.89 & $9.94^{* *}$ \\
\hline FBDO & $15.97^{a} \pm 0.83(14.75-17.54)$ & 5.20 & $16.05^{\mathrm{ab}} \pm 0.83(14.83-17.50)$ & 5.17 & $16.48^{b} \pm 0.54(15.75-17.38)$ & 3.28 & $4.11^{*}$ \\
\hline FBAN & $09.41^{\mathrm{ab}} \pm 0.67(08.37-10.48)$ & 7.17 & $09.60^{\mathrm{a}} \pm 0.82(08.59-11.72)$ & 8.51 & $08.99^{b} \pm 0.51(07.81-09.68)$ & 5.65 & $6.26^{* *}$ \\
\hline$L_{C P}$ & $19.02^{\mathrm{a}} \pm 1.01(17.03-20.65)$ & 5.33 & $17.69^{b} \pm 1.03(16.30-20.28)$ & 5.82 & $17.11^{b} \pm 0.69(16.42-19.27)$ & 4.01 & $33.96^{* *}$ \\
\hline CPD & $12.22^{\mathrm{a}} \pm 0.48(11.45-13.14)$ & 3.89 & $11.91^{b} \pm 0.53(10.47-13.04)$ & 4.49 & $11.82^{b} \pm 0.41(11.15-12.54)$ & 3.45 & $5.83^{* *}$ \\
\hline HD & $69.68^{\mathrm{a}} \pm 2.73(65.63-74.34)$ & 3.92 & $67.00^{b} \pm 2.93(62.25-75.67)$ & 4.37 & $65.31^{\mathrm{b}} \pm 2.68(60.37-70.02)$ & 4.10 & $18.91^{* \star}$ \\
\hline HW & $58.36^{\mathrm{a}} \pm 1.82(55.54-60.73)$ & 3.12 & $55.82^{b} \pm 1.15(53.49-58.79)$ & 2.06 & $56.46^{\mathrm{b}} \pm 1.68(53.72-60.08)$ & 2.98 & $21.11^{* *}$ \\
\hline MW & $25.48^{\mathrm{ab}} \pm 0.69(24.01-26.48)$ & 2.69 & $24.86^{\mathrm{a}} \pm 1.27(21.44-26.74)$ & 5.12 & $25.85^{\mathrm{b}} \pm 1.55(23.12-28.23)$ & 5.99 & $5.11^{* *}$ \\
\hline$L_{0}$ & $30.68^{\mathrm{a}} \pm 1.98(27.34-33.60)$ & 6.45 & $33.22^{\mathrm{b}} \pm 1.47(29.14-35.20)$ & 4.42 & $33.81^{b} \pm 1.15(31.41-35.11)$ & 3.40 & $33.58^{* *}$ \\
\hline$L_{\mathrm{PRO}}$ & $37.38^{\mathrm{a}} \pm 1.99(34.18-40.46)$ & 5.33 & $32.26^{b} \pm 1.74(28.10-36.18)$ & 5.38 & $31.87^{b} \pm 1.45(29.01-34.56)$ & 4.54 & $93.66^{* *}$ \\
\hline$L_{\mathrm{pO}}$ & $34.33^{\mathrm{a}} \pm 1.0^{\circ}(32.19-35.69)$ & 2.92 & $36.79^{b} \pm 2.67(33.91-42.37)$ & 7.27 & $37.69^{b} \pm 1.45(35.00-39.87)$ & 3.85 & $26.54^{\star *}$ \\
\hline$L_{\mathrm{MB}}$ & $26.68^{\mathrm{a}} \pm 2.33(22.94-32.38)$ & 8.75 & $29.84^{b} \pm 1.74(27.25-33.09)$ & 5.81 & $27.56^{\mathrm{a}} \pm 1.38(25.07-30.02)$ & 5.03 & $23.20^{* *}$ \\
\hline$C V p$ & & 5.36 & & 5.51 & & 4.91 & \\
\hline
\end{tabular}

For each morphometric variable, means with the same letter superscript are not significantly different. See table 2 for explanations of acronyms. *Significant at the $5 \%$ level ** Significant at the $1 \%$ level; NS, not significant at the $5 \%$ level; SD is standard deviation.

Table 4: Descriptive statistics of transformed morphometric variables, the Coefficient of Variation $(\mathrm{CV})$ of each measurement, the multivariate coefficient of variation of each species (CVp), and F-values (derived from the analysis of variance) of three populations of $P$. chalakkudiensis and similarly looking specimens, from South Kerala region of Western Ghats.

species, and also among populations of $P$. denisonii (CDR, KGD, VLP and CLR) and P. chalakkudiensis (CHD, PER and PMB), respectively. PCA of the 18 significant variables between $P$. chalakkudiensis and $P$. denisonii yielded 8 principal components, accounting for $82.23 \%$ of the total variation in the original variables. The variance explained by the first two components was found to be $48.63 \%$. The first component 
Citation: John L, Peter R, Gopalakrishnan A (2013) Population Structure of Denison's barb, Puntius denisonii (Pisces: Cyprinidae): A Species Complex Endemic to the Western Ghats of India. J Phylogen Evolution Biol 1: 106. doi:10.4172/2329-9002.1000106

Page 6 of 12

\begin{tabular}{|c|c|c|c|c|c|}
\hline & P. denisonii $(n=120)$ & & P. chalakkudiensis $(\mathrm{n}=90)$ & & \\
\hline & Mean \pm SD (min.-max.) & cV & Mean \pm SD (min.-max.) & CV & $F$ value \\
\hline$L_{\mathrm{s}}$ & $81.08 \pm 8.43(64.55-101.30)$ & - & $82.73 \pm 19.4^{\circ}(53.60-129.75)$ & - & - \\
\hline$L_{H}$ & $23.96 \pm 0.93(22.01-25.93)$ & 3.88 & $23.16 \pm 1.41(19.99-24.94)$ & 6.09 & $24.76^{* *}$ \\
\hline MBD & $24.42 \pm 1.23(21.96-26.41)$ & 5.04 & $26.83 \pm 1.0^{\circ}(24.93-29.30)$ & 3.73 & $229.33^{* \star}$ \\
\hline$L_{\mathrm{PRD}}$ & $47.12 \pm 1.03(44.96-49.51)$ & 2.19 & $46.91 \pm 1.4^{\circ}(44.51-50.81)$ & 2.98 & $1.59 \mathrm{NS}$ \\
\hline$L_{\mathrm{PD}}$ & $37.22 \pm 1.09(35.15-39.96)$ & 2.93 & $39.58 \pm 2.05(36.48-43.96)$ & 5.17 & $115.49^{* *}$ \\
\hline$L_{\mathrm{PRV}}$ & $52.99 \pm 2.01(49.19-58.95)$ & 3.79 & $50.47 \pm 1.65(47.01-53.56)$ & 3.26 & $93.74^{* *}$ \\
\hline$L_{\text {PRA }}$ & $75.55 \pm 1.69(72.31-78.88)$ & 2.24 & $74.09 \pm 1.55(70.17-77.25)$ & 2.09 & $41.49^{* \star}$ \\
\hline$L_{\text {PTFPLF }}$ & $28.1^{\circ} \pm 1.38(25.13-30.77)$ & 4.91 & $26.91 \pm 1.38(24.12-31.03)$ & 5.13 & $37.87^{* *}$ \\
\hline$L_{\text {PLFAF }}$ & $24.59 \pm 1.14(21.89-26.62)$ & 4.64 & $24.78 \pm 1.1^{\circ}(22.80-29.00)$ & 4.43 & $1.4^{\circ} \mathrm{NS}$ \\
\hline$L_{\mathrm{BC}}$ & $48.98 \pm 2.34(44.72-53.25)$ & 4.78 & $48.28 \pm 2.0^{\circ}(44.53-53.23)$ & 4.15 & $5.19^{*}$ \\
\hline FBDO & $16.41 \pm 0.63(15.18-18.45)$ & 3.84 & $16.17 \pm 0.77(14.75-17.54)$ & 4.78 & $6.13^{*}$ \\
\hline FBAN & $08.03 \pm 0.51(06.83-08.88)$ & 6.35 & $09.33 \pm 0.72(07.81-11.72)$ & 7.69 & $233.43^{* \star}$ \\
\hline$L_{C P}$ & $15.95 \pm 1.43(13.13-18.67)$ & 8.97 & $17.94 \pm 1.22(16.30-20.65)$ & 6.80 & $112.15^{\star \star}$ \\
\hline CPD & $10.99 \pm 0.51(10.27-12.65)$ & 4.64 & $11.98 \pm 0.5^{\circ}(10.47-13.14)$ & 4.18 & $196.12^{* \star}$ \\
\hline HD & $61.31 \pm 2.74(55.55-67.85)$ & 4.47 & $67.33 \pm 3.29(60.37-75.67)$ & 4.89 & $208.39^{* *}$ \\
\hline HW & $55.8^{\circ} \pm 2.45(50.77-61.95)$ & 4.39 & $56.88 \pm 1.9^{\circ}(53.49-60.73)$ & 3.34 & $12.11^{* *}$ \\
\hline MW & $26.22 \pm 2.01(22.60-31.23)$ & 7.67 & $25.4^{\circ} \pm 1.28(21.44-28.23)$ & 5.03 & $11.43^{\star \star}$ \\
\hline$L_{0}$ & $31.45 \pm 1.6^{\circ}(28.56-34.22)$ & 5.09 & $32.57 \pm 2.07(27.34-35.20)$ & 6.34 & $19.65^{\star *}$ \\
\hline$L_{\text {PRO }}$ & $32.59 \pm 1.67(28.97-35.88)$ & 5.12 & $33.84 \pm 3.05(28.10-40.46)$ & 9.03 & $14.37^{* *}$ \\
\hline$L_{\mathrm{PO}}$ & $38.39 \pm 1.82(34.28-41.94)$ & 4.74 & $36.27 \pm 2.32(32.19-42.37)$ & 6.40 & $55.22^{* *}$ \\
\hline$L_{\text {MB }}$ & $32.06 \pm 3.44(22.56-38.31)$ & 10.73 & $28.03 \pm 2.28(22.94-33.09)$ & 8.12 & $93.00^{\star \star}$ \\
\hline$c V p$ & & 5.79 & & 5.85 & \\
\hline
\end{tabular}

Table 5: Descriptive statistics of transformed morphometric variables, the Coefficient of Variation (CV) of each measurement, the multivariate coefficient of variation of each species (CVp) and F-values (derived from the analysis of variance) of $P$. denisonii and $P$. chalakkudiensis.

was mainly defined by measurements of $L_{\mathrm{PRD}}$, CPD, $L_{\mathrm{CP}}$, MBD, $L_{\mathrm{PD}}$ and FBAN. The second component was mainly correlated with $L_{\text {PTPPLF }}, L_{\text {PRA }}, \mathrm{HD}$ and $L_{\text {PRV }}$. These observations indicated that the above morphometric characters contributed maximum in differentiating $P$. denisonii and P. chalakkudiensis. The scatter diagram based on PCA clearly distinguishes the two major groups, which are evidently distinct as two species (Figure 4a), (Supplementary data (Table 9)).

Out of 20 morphometric measurements (excluding $L_{\mathrm{s}}$ ) taken, 18 measurements found to be significant $(\mathrm{p}<0.05)$ among the populations of $P$. denisonii, and 19 measurements found to be significant $(\mathrm{p}<0.05)$ among the populations of $P$. chalakkudiensis. These significant morphometric variables were used to carry out PCA with the population samples of both species. Eight principal components accounted to describe $80.39 \%$ of the total variation in P. denisonii and $81.99 \%$ in $P$. chalakkudiensis in the original variables. Among P. denisonii population data, the first two components explained $46.33 \%$ variance, in which the first component was mainly defined by measurements of FBDO, $L_{\text {PTFPLF }}$ HD, $L_{\mathrm{PRA}}$ and $L_{\mathrm{PO}}$. The second component was mainly correlated with the measurements of $L_{\mathrm{MB}}, L_{\mathrm{CP}}, L_{\mathrm{PLFAF}}, L_{\mathrm{H}}$ and FBAN. This indicated that the above morphometric characters contributed maximum in differentiating $P$. denisonii populations. The bivariate scatter plot of component 1 and 2 was found to be sufficient to outline the morphological heterogeneity existing among $P$. denisonii populations (Figure 4b) (Supplementary Data (Table 10)). The first two principal components explained about $43.54 \%$ variance of total variation in the original variables in P. chalakkudiensis. The first component was mainly defined by $L_{\mathrm{PD}}, L_{\mathrm{CP}}, L_{\mathrm{PRV}}, L_{\mathrm{O}}$ and $L_{\mathrm{PRO}}$; and the second component was mainly correlated with length of $L_{\mathrm{PLFAF}}, L_{\mathrm{PTFPLF}}, L_{\mathrm{MB}}, L_{\mathrm{BC}}$ and $L_{\mathrm{PO}}$. These indicated that the above morphometric characters contributed the maximum to differentiate $P$. chalakkudiensis populations. The bivariate scatter plot of component 1 and 2 was found to be sufficient to outline the morphological heterogeneity existing among populations of $P$. chalakkudiensis (Figure 4c) (Supplementary Data (Table 11)).

\section{Sequence characteristics, genetic divergence and population variability}

An 832 bp DNA sequence comprising partial regions of ATP synthase 8 and 6 genes were studied from 74 samples, including $P$. denisonii and $P$. chalakkudiensis from seven different geographic locations. Out of the 832 characters obtained, $676(81.25 \%)$ were constant and $156(18.75 \%)$ were variable, in which 144 (17.31\%) were informative for parsimony among all population samples, including $P$. denisonii and P. chalakkudiensis. Sequence comparison revealed 32 different haplotypes, defined by 156 divergent nucleotide sites. DNA sequences of all different haplotypes are deposited in GenBank (accessions GQ247534 to GQ247548 and JF927912 to JF927928). Among the 7 different river systems analyzed, the Group A $(P c)$, samples were characterized by the presence of a single haplotype of high frequency, accompanied by several other closely related haplotypes of lower frequency. The CHD and PER(c) samples were represented by the high frequency haplotype, and the accompanying low frequency haplotypes were distributed again in PER(c) and PMB populations (Table 6). The sample population of Chalakkudy river (CHD) of $P$. chalakkudiensis was found to be the least diverse based on haplotype diversity ( 0.0$)$ observed. The Periyar river system showed the maximum number of haplotypes ( 9 out of 17 samples sequenced), which included both morphotypes resembling the morphologic features of $P$. denisonii and $P$. chalakkudiensis. Haplotype diversity $(h)$, within the geographic populations, was high in the case of Group B $(P d)$ samples [ranged from 0.2500 in KGD to 0.9643 in PER(d)], than that observed within Group A $(P c)$ samples (ranged from 0.0 in CHD to 0.8462 in $\mathrm{PMB})$; whereas the nucleotide diversity $(\pi)$ was generally low and ranged from $0.0(\mathrm{CHD})$ to 0.0056 [PER(d)]. Except CHD and PER(c), none of the population samples shared common haplotypes, indicating significant genetic separation among different populations. The only single haplotype (H17) observed within CHD was found to be shared with PER(c) (Table 6). 

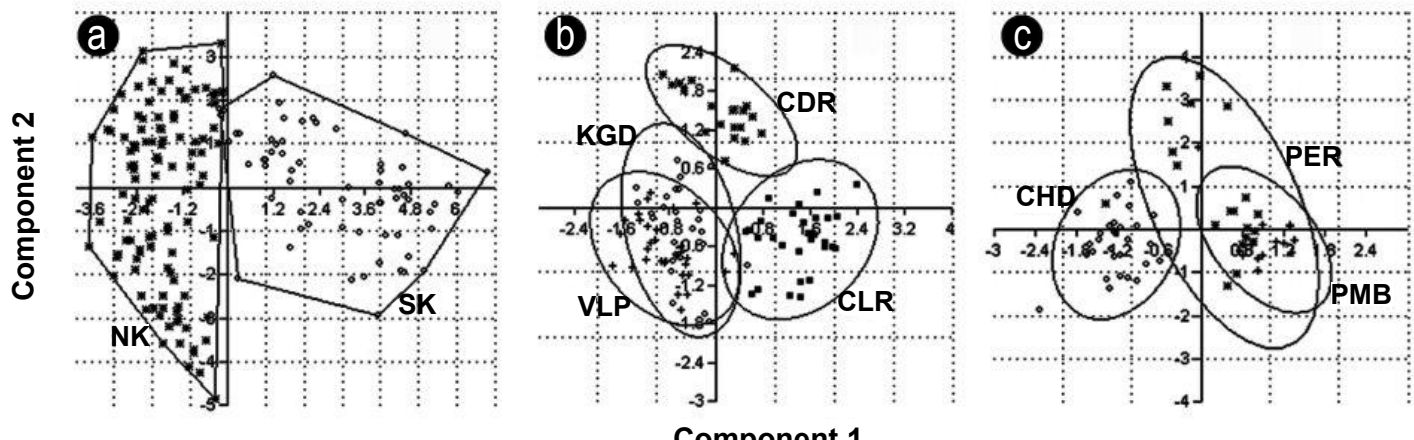

Component 1

Figure 4: Scatter plots of principal component 1 and principal component 2 from PCA of significant morphometric variables from $P$. denisonii and $P$. chalakkudiensis specimen from different isolated water sheds; a-With the specimens of $P$. denisonii from North Kerala and Karnataka region (NK includes CDR, KGD, VLP and CLR), and that of Central and South Kerala region (SK includes CHD, PER and PMB; P. chalakkudiensis and its look alkies') of the Western Ghats were analyzed as two groups; b-With four different populations from North Kerala and Karnataka (CDR, KGD, VLP and CLR) region analyzed separately; c-With three different populations from Central and South Kerala (CHD, PER and PMB) region analyzed separately.

\begin{tabular}{|c|c|c|c|c|c|c|c|c|}
\hline & \multicolumn{5}{|c|}{ P. denisonii } & \multicolumn{3}{|c|}{ P. chalakkudiensis } \\
\hline & CDR & KGD & VLP & CLR & PER(d) & PER(c) & CHD & PMB \\
\hline CDR & $0.17 \%$ & $0.9686(+)$ & $0.9390(+)$ & $0.9761(+)$ & $0.9577(+)$ & $0.9901(+)$ & $0.9913(+)$ & $0.9843(+)$ \\
\hline KGD & $0.0349(0.0081)$ & $0.03 \%$ & $0.7647(+)$ & $0.9910(+)$ & $0.9644(+)$ & $0.9972(+)$ & $0.9986(+)$ & $0.9890(+)$ \\
\hline VLP & $0.0299(0.0162)$ & $0.0039(0.0770)$ & $0.21 \%$ & $0.9756(+)$ & $0.9537(+)$ & $0.9891(+)$ & $0.9905(+)$ & $0.9829(+)$ \\
\hline CLR & $0.0537(0.0061)$ & $0.0617(0.0023)$ & $0.0578(0.0063)$ & $0.07 \%$ & $0.9611(+)$ & $0.9950(+)$ & $0.9963(+)$ & $0.9874(+)$ \\
\hline PER(d) & $0.0834(0.0111)$ & $0.0862(0.0092)$ & $0.0859(0.0121)$ & $0.0795(0.0101)$ & $0.56 \%$ & $0.9686(+)$ & $0.9702(+)$ & $0.9658(+)$ \\
\hline PER(c) & $0.1146(0.0025)$ & $0.1128(0.0007)$ & $0.113^{\circ}(0.0028)$ & $0.109^{\circ}(0.0013)$ & $0.0924(0.0081)$ & $0.03 \%$ & $0.0000(-)$ & $0.7510(+)$ \\
\hline CHD & $0.1145(0.0022)$ & $0.1128(0.0003)$ & $0.1129(0.0024)$ & $0.1089(0.0009)$ & $0.0923(0.0307)$ & 0.0000 & $0.0 \%$ & $0.7822(+)$ \\
\hline PMB & $0.1193(0.0040)$ & $0.1175(0.0028)$ & $0.1177(0.0044)$ & $0.1136(0.0032)$ & $0.0969(0.0088)$ & $0.0034(0.0829)$ & $0.0037(0.0696)$ & $0.17 \%$ \\
\hline
\end{tabular}

$(+)$, significant at $p<0.05$ after sequencial Bonferroni agjustment; (-), not significant at this level; (c), PER specimen with a black blotch on dorsal fin; (d), PER specimen without a black blotch on dorsal fin

Table 6: Mean pairwise distances, gene flow $(\mathrm{Nm})$ and Pairwise $F_{\mathrm{sT}}$ among populations of $P$. denisonii and $P$. chalakkudiensis, based on $A T P a s e$ 8/6 gene sequences. Pairwise $F_{s,}$ and its significance in parenthesis, are given above diagonal; genetic distance based on $\mathrm{K} 2 \mathrm{P}$ and $\mathrm{Nm}$ in parenthesis is given below diagonal; mean $\mathrm{K} 2 \mathrm{P}$ distance within each population is given in $\%$ across the table.

The mean K2P distances and pairwise $F_{S T}$ values observed between populations across $P$. denisonii and $P$. chalakkudiensis were found to be markedly higher than that observed within each population (Table 7). Mean K2P distances observed between the Group A $(P c)$ and Group $\mathrm{B}(P d)$ population samples ranged from 0.0969 (between PER $(\mathrm{d})$ and $\mathrm{PMB}$ ) to 0.1193 (between CDR and PMB)). Distance observed among samples of Group B $(P d)$ was comparatively higher, whereas that among samples of Group A $(P c)$ were markedly smaller (Table 7). The PER(d) population was found to be equally separated from all other populations, with mean $\mathrm{K} 2 \mathrm{P}$ distances values ranging from 0.0795 (with CLR) to 0.0969 (with PMB). The pattern of genetic diversity observed was moderate to high, and nucleotide diversity was low in general within the populations of $P$. denisonii and $P$. chalakkudiensis (Table 6).

Analysis of molecular variance (Table 8), for the two species as groups, indicated a total variance of $65.31 \%\left(\Phi_{\mathrm{CT}}=0.653\right)$, which was significant $(P<0.05)$ enough, and attributed to the differences between them. Grouping individuals into 3 or 4 groups based on morphologic features and $\mathrm{K} 2 \mathrm{P}$ distances observed further emphasized significant $(P<0.005 ; 0.001)$ genetic divergence among the geographically isolated populations of $P$. denisonii.

\section{Phylogenetic relationships}

Phylogenies constructed using the Maximum Likelihood, Maximum parsimony (Figures $5 \mathrm{a}$ and $\mathrm{b}$ ), and Neighbor Joining (not presented in figure) methods showed similar topologies, and consistently indicated clear population structuring across geographically distinct riverine populations of $P$. denisonii. All specimens of Group B $(P d)$ formed four well differentiated monophyletic sister groups (clades), as clade 1 (formed by CDR population samples), clade 2 (formed by VLP and KGD samples), clade 3 (formed by CLR samples) and clade 4 (formed by PER(d) samples, the specimens without a black blotch on dorsal fin obtained from the river Periyar)-all with high bootstrap support; whereas in the case of Group A $(P c)$, all specimens of $P$. chalakkudiensis from its type locality, Chalakkudy river (CHD) and from the other 2 different river systems-Periyar [PER(c)] and Pamba (PMB) were found to be clustered together into an unresolved sister clade, indicating least genetic divergence among the riverine populations of the species.

\section{Discussion}

\section{Biometric and genetic divergence in $P$. denisonii complex}

The present study reveals clear distinction between the two closely related species, $P$. denisonii and $P$. chalakkudiensis. The presence of high level of genetic divergence among geographically separated populations of $P$. denisonii suggests the possibility of cryptic species within them. Day [4] described the species P. denisonii in his work "On the fishes of Cochin on the Malabar cost of India", as Labeo denisonii, obtained in the hill ranges of Travancore. Later, in his works on the 
Citation: John L, Peter R, Gopalakrishnan A (2013) Population Structure of Denison's barb, Puntius denisonii (Pisces: Cyprinidae): A Species Complex Endemic to the Western Ghats of India. J Phylogen Evolution Biol 1: 106. doi:10.4172/2329-9002.1000106

Page 8 of 12

\begin{tabular}{|c|c|c|c|c|}
\hline Population & $\boldsymbol{n}_{\boldsymbol{t}}$ & $\boldsymbol{n}_{\boldsymbol{h}}$ & Haplotypes at each sampling site $\left(\boldsymbol{n}_{\boldsymbol{h}}\right)$ & Nucleotide diversity (m) \\
\hline CDR & 10 & 5 & $\mathrm{H} 01(2), \mathrm{H} 02(1), \mathrm{H} 03(5), \mathrm{H} 04(1), \mathrm{H} 05(1)$ & 0.0017 \\
\hline KGD & 8 & 2 & $\mathrm{H} 06(7), \mathrm{H} 07(1)$ & 0.0003 \\
\hline VLP & 8 & 6 & $\mathrm{H} 08(1), \mathrm{H} 09(3), \mathrm{H} 10(1), \mathrm{H} 11(1), \mathrm{H} 12(1), \mathrm{H} 13(1)$ & 0.0021 \\
\hline CLR & 9 & 3 & $\mathrm{H} 14(7), \mathrm{H} 15(1), \mathrm{H} 16(1)$ & 0.0007 \\
\hline PER(d) & 8 & 7 & $\mathrm{H} 19(1), \mathrm{H} 20(2), \mathrm{H} 21(1), \mathrm{H} 22(1), \mathrm{H} 23(1), \mathrm{H} 24(1), \mathrm{H} 25(1)$ & 0.8929 \\
\hline PER(c) & 9 & 2 & $\mathrm{H} 17(8), \mathrm{H} 18(1)$ & 0.4167 \\
\hline CHD & 9 & 1 & $\mathrm{H} 17(9)$ & 0.9643 \\
\hline PMB & 13 & 7 & $\mathrm{H} 26(2), \mathrm{H} 27(5), \mathrm{H} 28(1), \mathrm{H} 29(1), \mathrm{H} 30(1), \mathrm{H} 31(2), \mathrm{H} 32(1)$ & 0.0003 \\
\hline
\end{tabular}

${ }^{*}, P<0.05 ;{ }^{* *}, P<0.01 ;{ }^{* * *}, P<0.005 ; n_{t}$-Number of individuals analyzed per site; $n_{n}$-Number of haplotypes per site

Table 7: Intrapopulation nucleotide $(\pi)$ and haplotype $(h)$ diversities per population of $P$. denisonii and $P$. chalakkudiensis based on ATPase $8 / 6$ gene sequences.

\begin{tabular}{|c|c|c|c|c|c|}
\hline Grouping tested & Source of variation & $d f$ & $\%$ total variance & $\Phi$ & $P$-value \\
\hline \multirow{3}{*}{$\begin{array}{l}\text { Group } 1 \\
\text { [CDR+KGD+VLP+CLR+PER(d)] } \\
\text { Group } 2[C H D+P E R(c)+P M B]\end{array}$} & Among groups & 1 & 65.31 & $\Phi_{C T}=0.653$ & $<0.05$ \\
\hline & Among populations within groups & 6 & 33.25 & $\Phi_{\mathrm{SC}}=0.958$ & $<0.001$ \\
\hline & Within populations & 66 & 1.44 & $\Phi_{\mathrm{ST}}=0.986$ & $<0.001$ \\
\hline \multirow{3}{*}{$\begin{array}{l}\text { Group } 1[\mathrm{CDR}+\mathrm{KGD}+\mathrm{VLP}] \\
\text { Group } 2[\mathrm{CLR}+\mathrm{PER}(\mathrm{d})] \\
\text { Group } 3[\mathrm{CHD}+\mathrm{PER}(\mathrm{c})+\mathrm{PMB}]\end{array}$} & Among groups & 2 & 72.54 & $\Phi_{\mathrm{CT}}=0.725$ & $<0.005$ \\
\hline & Among populations within groups & 5 & 25.81 & $\Phi_{\mathrm{SC}}=0.940$ & $<0.001$ \\
\hline & Within populations & 66 & 1.65 & $\Phi_{\mathrm{ST}}=0.983$ & $<0.001$ \\
\hline \multirow{3}{*}{$\begin{array}{l}\text { Group } 1 \text { [CDR+KGD+VLP] } \\
\text { Group } 2 \text { [CLR ] } \\
\text { Group } 3[\text { [PER(d)] } \\
\text { Group } 4 \text { [CHD+PER(c)+PMB] }\end{array}$} & Among groups & 3 & 85.16 & $\Phi_{\mathrm{CT}}=0.852$ & $<0.001$ \\
\hline & Among populations within groups & 4 & 13.20 & $\Phi_{S C}=0.890$ & $<0.001$ \\
\hline & Within populations & 66 & 1.64 & $\Phi_{\mathrm{ST}}=0.984$ & $<0.001$ \\
\hline
\end{tabular}

Table 8: Results of the hierarchical analysis of molecular variance (AMOVA) of populations of $P$ denisonii and $P$ chalakkudiensis, based on mitochondrial ATPase 8/6 region. Significance values were obtained from $100^{\circ}$ random permutations of the DNA sequence using Arlequin 3.0.

"Fishes of Malabar" and "The Fishes of India; being a Natural History of the fishes known to inhabit the seas and freshwaters of India, Burma and Ceylon", he described the species as Puntius denisonii and Barbus denisonii, respectively. Presently the valid generic name is Puntius. According to Menon et al. [1], P. chalakkudiensis can be distinguished from $P$. denisonii and all other Puntius species by its inferior mouth and a distinct black blotch on the dorsal fin. In the present study, a series of phenotypic characters [ 18 out of 20 morphometric characters-other than $L_{\mathrm{S}}$-examined (Table 5)] showed significant differences revealed by the univariate Analysis of Variance (ANOVA), to differentiate $P$. denisonii and $P$. chalakkudiensis. The multivariate analysis PCA using significant morphometric variables clearly indicated the separation of two species, without any inter mixing (Figure 4a). Structural variation observed on examination of gill rakers on the first gill arch was distinct between the two species (Figure 3). The color pattern observed in the adult specimens of $P$. denisonii, $P$. chalakkudiensis and their juveniles were also different (Figure 2), which emphasized the clear differentiation of $P$. chalakkudiensis from $P$. denisonii. Breeding season of $P$. denisonii was reported during the month of October to March [45], however, a slight variation in the peak breeding months among different watershed populations within the species was observed.

Molecular analysis based on ATPase 8 and 6 genes showed unambiguous genetic divergence between the two species. Both species were genetically distinct, as the amount of pairwise sequence divergence within them was much less than that between the two (Table 7). The degree of genetic divergence between the two species was comparable to that existing between many of the teleost species [46], including several cyprinid species from the Western Ghats [47]. In the phylogenetic analysis based on both ML, MP (Figure 5) and NJ methods, all the haplotypes with a black blotch on dorsal fin (CHD, PER(c) and PMB population samples), which is a typical characteristic of $P$. chalakkudiensis, formed a single monophyletic clade, well separated from the other sister clades. Based on the morphologic and genetic pattern observed in the present study, the specimens with a black blotch on dorsal fin obtained from Chalakkudy, Periyar and Pamba river systems (CHD, PER(c) and PMB samples, respectively), could be considered as $P$. chalakkudiensis. Thus, the distribution of P. chalakkudiensis should be extended to Periyar and Pamba River systems, in addition to the previously reported type locality, the Chalakkudy River [1] and Achankovil [48]. The rest of the haplotypes of $P$. denisonii, without a black blotch on dorsal fin, obtained from four different river systems of north Kerala (CDR, KGD, VLP and CHR samples) and from Periyar river [PER(d)], were further separated into four distinct well separated monophyletic clades (C1, C2, C3 and C4), with high level of genetic (Figure 5; Table 7), and morphologic divergence (Figure $4 \mathrm{~b}$; Table 3 ), indicating the presence of four independent evolutionary lineages within the species. An unrecognized diversity and possibility of at least two morphologically cryptic lineages within $P$. denisonii, obtained from international aquarium trade, has been reported recently [46].

The species $P$. denisonii and $P$. chalakkudiensis have been reported from different west flowing rivers of Kerala [1-3,48], including the river systems under the present study. Earlier reports regarding the distribution of these species were purely based on the morphological examinations alone. The Periyar river system was found to be the most diverse, based on the pattern of haplotype diversity observed (Table 6), and more over with the presence of two morphotypes [PER(d) and PER(c)], which resemble the characteristics of $P$. denisonii and $P$. chalakkudiensis, respectively. Even though the specimens without a black blotch [PER(d)], obtained from the same habitat, resembled $P$. denisonii in many features, especially the shape of the head, position of mouth and a more pointed snout, the colour pattern shows more similarity with $P$. chalakkudiensis (Figure 2c). The pattern of clustering with phylogenetic analysis (Figure 5 ) and the mean pairwise distances value (0.0924) observed between the two morphotypes [PER(d) and PER(c)], obtained from the Periyar river system, clearly indicates them as two genetically distinct groups, as members of the two different species. The molecular data revealed the PER(d) population samples are almost equally well separated from the North Kerala specimens of $P$. denisonii (mean pairwise distance value ranged from 0.0795 with CLR to 0.0862 with KGD), and the typical $P$. chalakkudiensis (mean pairwise GD 0.0923; Table 7 and Figure 5). The mean K2P distance observed 

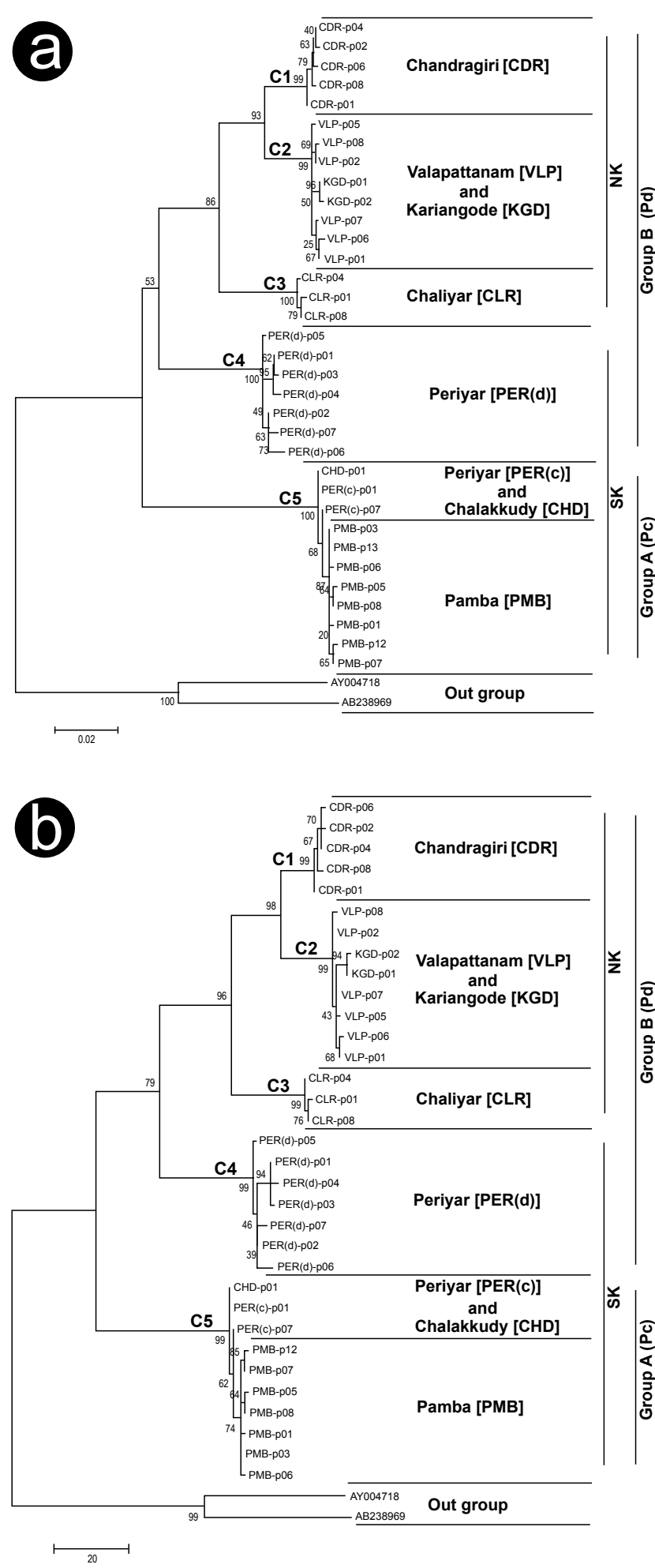

Figure 5: (a) Maximum Likelihood and (b) Maximum Parsimony trees of the populations of $P$. denisonii and $P$. chalakkudiensis inferred from haplotype (representative haplotypes alone showed here) sequence variation of the mitochondrial DNA ATPase $8 / 6$ genes. Numbers at nodes indicate the bootstrap values. AY004718 (Puntius conchonius) and AB238969 (Puntius ticto) are from Genbank, and are included as outgroup species. between the samples of $P$. denisonii and P. chalakkudiensis was found to be significantly higher ranging from 0.0923 (between PER(d) and CHD) to 0.1193 (between CDR and PMB). Even though, the type locality from where Francis Day described P. denisonii was mentioned as Mundakayam, Travancore hill region of the Western Ghats [4], any specific stream or river system has not been mentioned anywhere. The sampling survey carried out in and around Mundakayam during the present study could obtain specimens of Denison's barb from the river Pamba. The sampling site at Pamba River was about $15 \mathrm{~km}$ away from Mundakayam town, and the specimens obtained resembled the typical $P$. chalakkudiensis. But, these specimens obtained near the type locality in the present study could not be considered as the topotypes of typical P. denisonii. Because, on examination it is observed that the type series of $P$. denisonii deposited in the Natural History Museum, London, is devoid of a clear black blotch on the dorsal fin, and with terminal mouth (personal communication, Dr. K. Rema Devi, Zoological Survey of India and Dr. Rajeev Raghavan, Conservation Research Group, Kerala, India). However, though we could not obtain a single specimen without a black blotch on dorsal fin (typical P. denisonii) from Pamba, the chances for the occurrence of such specimens in those habitats towards South Kerala region could not be eliminated.

The present mtDNA locus ATPase 8 and 6 genes studied showed almost similar genetic divergence (K2P) and phylogenetic patterns observed with the DNA barcoding locus COI, within the species $P$. denisonii [49]. However, the pattern of monophyletic association exhibited among different lineages within the species $P$. denisonii, with high branch support, and with an average of $10 \mathrm{X}$ greater divergence between than within each lineage (Figure 5, Table 7) satisfies the basic concept of COI based DNA barcoding, for species identification and discovery [50-52], in identifying new species. The high level of genetic and morphologic diversity observed among the Denison's barb, distributed along the isolated habitats of the Western Ghats, make it a species complex, with a scope of at least four cryptic species within them, being discussed further. The population samples such as CDR, CLR and PER(d) were found to be well separated from VLP and KGD samples of $P$. denisonii (Figure 5, Table 7). A thorough study with detailed sampling from all the distributed areas of these species, and comparison with available data on type specimen, is required to get a conclusive picture regarding the taxonomic positions of the species.

\section{Population structure}

Freshwater fish are expected to display greater levels of genetic differentiation and population subdivision than marine species, due to the isolating nature of river systems and small effective population sizes [53,54]. Due to the rugged landscape, most rivers of the Western Ghats region are separated from each other by mountain ridges at medium and high elevations, where these species $(P$. denisonii and $P$. chalakkudiensis) are distributed. Generally, neighboring rivers are not connected to each other, and substantial genetic subdivision among populations was expected. In the present study, the mtDNA data reveal that the levels of genetic differentiation among population samples of $P$. denisonii between drainages were consistent with these expectations.

Univariate and multivariate analyses of morphometric traits clearly demonstrated an intraspecific morphological variation among the samples of $P$. denisonii and P. chalakkudiensis, from different River systems of the Western Ghats of India (Tables 3 and 4, Figure 4). Measurements of head were the most discriminating variable in this study. Accordingly, the samples from Chaliyar River possess the deepest head and lowest $L_{\mathrm{PO}}$, whereas the Chandragiri population possesses the highest mean $L_{\mathrm{H}}$ (Table 3). But, in the case of P. chalakkudiensis, the 
variation among population samples was mainly related to measures of $L_{\mathrm{PRO}}, \mathrm{HW}, L_{\mathrm{PRV}}, L_{\mathrm{O}}, L_{\mathrm{PD}}$, and $L_{\mathrm{PO}}$. The coefficient of variation observed in the present study was comparatively low across all the populations studied, ranging from $4.80 \%$ (CDR) to $5.51 \%$ (PER). In fishes, the coefficients of variation within populations are usually far greater than $10 \%$ [55]. The lower coefficient of variation indicates minimal or very low intra-population variation. Similar results were obtained by Mamuris et al. [56], in the seven populations of red mullet (Mullus barbatus) and by Quilang et al. [27], in four populations of Silver perch (Leiopotherapon plumbeus).

Environmental factors, in particular the temperature that prevails during some of the sensitive developmental stages, can have great influence on the morphological characters [57,58]. Intraspecific morphological heterogeneity observed in the present study may explain the effect of phenotypic plasticity, as the genetic data do not allow the rejection of the null hypothesis of panmixia for P. chalakkudiensis, especially between CHD and PER(c). The physico-chemical parameters could have an effect on the development of $P$. chalakkudiensis, although studies on reproductive, as well as foraging behavior, especially during its larval stage, are yet to be conducted. Many teleost populations exhibit morphological changes depending on the environment, and are still genetically homogenous, as reported in Brazilian populations of yellowtail snapper (Ocyurus chrysurus, Lutjanidae) [59], and in three different riverine populations of Liza $a b u$, a mugilid species from Turkey [60]. However, in the present study, it may be difficult to point out which of the several biotic and abiotic factors contributed to this. But, the morphological heterogeneity observed in P. denisonii populations is highly consistent with the genetic data, and most probably does not result from phenotypic plasticity. The present study has thus, shown the efficiency of using multivariate techniques in analyzing the population structure, in both species. However, the true reasons for the observed morphologic variability needs further studies, extended to other watersheds also, if the natural distribution of the species exists there.

Analysis of molecular variance, F-statistics and phylogenetic analysis indicated marked genetic structure in P. denisonii populations at the inter-regional scale. The extent of genetic differentiation observed among the populations of $P$. denisonii is related to the degree of geographic separation between river systems, from where the samples were collected. The ANOVA analyses showed that populations from different regions were differentiated from each other (Table 8). A significant $\Phi_{\mathrm{SC}}$ value indicated that a major amount of the variance could be ascribed to among population divergence. The pair wise $F_{\mathrm{ST}}$ (Table 7) values derived the same conclusion, which suggests that genetic differentiation can be largely explained by the limited dispersal of $P$. denisonii, among regions due to geographical barriers. Space is not the only parameter that determines genetic population structure and gene flow. Instead, landscape features between populations can influence dispersal rates and migration success [61]. The degree of differentiation between populations is obviously associated with highly rugged geographical pattern of the Western Ghats, leading to the formation of fragmented populations of the species with limited dispersal abilities. The reconstructed phylogenetic trees, as seen from this study, also supported the high differentiation among geographical regions. In the present study, the $\mathrm{Nm}$ values observed were considerably low; indicating that gene flow among spatially distant populations of both the species is very restricted (Table 7). In fact, a value of $\mathrm{Nm}$ much lower than 1 is interpreted as evidence of gene flow, insufficient in preventing differentiation, as a result of genetic drift $[62,63]$. None of the haplotypes were found to be shared between any population samples analyzed in the present study, other than Periyar and Chalakkudy samples of $P$. chalakkudiensis. This could represent occasional or rare migrants between the later. The overall level of genetic exchange may be below that required to homogenize populations, or there may be a barrier to gene flow between these populations. It may also be noted that phylogenetic analysis revealed separate clades of haplotypes, representing each population. This suggests some degree of reproductive isolation of $P$. denisonii populations, leading to geographical structuring with limited or no gene flow between the populations.

The pattern of genetic diversity with high genetic variation and low to moderate nucleotide diversity may be attributed to a population expansion after a low effective population size, caused by 'bottlenecks' or 'founder events' [64]. In such cases, the rapid growth of a population leads to the retention of new mutations, especially in mitochondrial DNA sequences that is known to evolve several times faster than nuclear DNA [65]. Such patterns of diversity strongly suggest a historical influence on the genetic structure of populations, as estimated by analysis of haplotype frequencies in $P$. denisonii population samples (Table 6). Similar pattern of mtDNA variation has been observed in another highly endangered cyprinid species, Anaecypris hispanica, which is endemic to the Guadiana River basin in the Iberian Peninsula [66].

Both $P$. denisonii and $P$. chalakkudiensis have shown a marked decline in abundance and population in recent years [67-69]. Intensive collection efforts and fishing from the wild to supply the demand for the species in aquarium trade, and the lack of commercial hatchery or captive breeding technology, together contributed to the decline of abundance and population. This could have an important direct influence on genetic variation, as populations with lower effective sizes are more affected by random genetic drift, and gene flow tends to decrease. Populations with smaller effective population sizes also tend towards rapid fixation of haplotypes, which might be one of the reasons behind the reduced number of haplotypes found in $P$. chalakkudiensis population, and higher number of unique haplotypes found in $P$. denisonii populations.

\section{Phylogenetic relationships}

The results of phylogenetic analyses in the present study: 1) establish the validity of $P$. chalakkudiensis and $P$. denisonii as distinct species; 2) estimate the validity of subpopulations within $P$. denisonii; 3) clarify the relationships of populations of $P$. denisonii, with that of $P$. chalakkudiensis, and 4) indicate the presence of different evolutionary lineages forming cryptic species within P. denisonii.

A monophyletic pattern was observed with all the analyses. All haplotypes of the two species separated clearly as two major distinct groups, thus exhibiting phylogenetic separation between P. chalakkudiensis (Group A) and P. denisonii (Group B). A distinct pattern could be visualized within group $B$, as four well separated clades $(\mathrm{C} 1, \mathrm{C} 2, \mathrm{C} 3$ and $\mathrm{C} 4)$ reflecting a clear region-wise population sub-structuring. Even though clade C2 is formed by VLP and KGD populations, some degree of isolation could be observed, without any shared haplotypes among them. Overall, there was no evidence of homogeneity among these populations; support for some degree of structuring existing among populations of $P$. denisonii, based on nucleotide data and geographical nature of the site. However, no distinct sub-structuring was observed for CHD and PER(c) populations of $P$. chalakkudiensis; PMB was found to be separated from them without any shared haplotypes. 
Citation: John L, Peter R, Gopalakrishnan A (2013) Population Structure of Denison's barb, Puntius denisonii (Pisces: Cyprinidae): A Species Complex Endemic to the Western Ghats of India. J Phylogen Evolution Biol 1: 106. doi:10.4172/2329-9002.1000106

Page 11 of 12

\section{Implications for fisheries management and conservation}

Puntius denisonii and P. chalakkudiensis are exploited in large numbers from the Western Ghats, to meet the demand from domestic and international aquarium trade [7], and as such the issue of population structure/gene flow is critical to their conservation and management. The result of the present study strongly indicates that, although both species appear similar in some respects, management strategies should be developed for each species separately. Findings of the present study have direct relevance to the definition of conservation units for these species [70]. Under this criterion, the five riverine populations of $P$. denisonii, viz. Chandragiri, Kariangode, Valapattanam, Chaliyar and Periyar (d), and two populations of $P$. chalakkudiensis (Chalakkudy and Periyar (c) together forms one, and Pamba), can be proposed as distinct MUs, which require separate monitoring and management. In future, a conservation policy based on these MUs is advisable. The genetic data further suggest the presence of at least four evolutionarily significant units, ESUs [70], within P. denisonii: (1) Chandragiri (2) Valapattanam and Kariangode (3) Chaliyar and (4) Periyar(d). These groups have been isolated and represent evolutionarily independent lineages in all phylogenetic analysis, and the gene flow between the groups is restricted.

In contrast, the analysis for $P$. chalakkudiensis shows a very different picture. The lack of genetic divergence found in this species, especially between the Chalakkudy and Periyar populations, is consistent with a "one stock" management; whereas with Pamba population, it exhibits a high level of genetic divergence, indicating a population expansion after a bottleneck. Secondly, the extremely low level of genetic variation observed within this species suggests that the population as a whole has undergone a dramatic reduction in size, in the recent evolutionary past. As a consequence, the reduced level of variation could compromise the 'genetic health' of the population, and thus may affect its long term viability. Efforts should be directed to ensure that the current level of genetic variation for this species is not eroded further. Hence, conservation action should concentrate on both species separately, especially as the species $P$. chalakkudiensis appears to have a smaller population size than $P$. denisonii. Habitat protection for natural breeding would be a solution to sustain the subpopulations within each region, in which these species are found.

\section{Acknowledgements}

The authors wish to thank the Director, NBFGR, Lucknow for providing the facilities for undertaking this work. The critical comments, suggestions and guidance provided by Dr. E.G. Silas (former Vice Chancellor of Kerala Agricultura University, Kerala), during the course of this study, is greatly acknowledged. Thanks to Dr. K.K. Vijayan (Head, Marine Biotechnology Division, CMFRI, Kochi) for providing all the facilities to complete the molecular analysis of the present study. The authors also thank Basheer V.S. and Siby Philip (NBFGR Cochin Unit, Kochi), AnnaMercy T.V. and Dinesh K. (College of Fisheries, Kochi), for their help during the collection of samples; Jeena N.S., Vidya R. and Sajeela K.A. (NBFGR Cochin Unit, Kochi), Vikas P.A. and Subin C.S. (CMFRI, Kochi), for their kind help in lab during the study; Ratheeshan K and Ambrose T.V. (CMFRI, Kochi), for their help in carrying out the statistical analysis of morphometric data; Manoj P. (RGCB, Thiruvananthapuram), for his help in sequencing some of the DNA samples.

\section{References}

1. Menon AGK, Rema Devi K, Thobias MP (1999) Puntius chalakkudiensis, a new colourful species of Puntius (Family: Cyprinidae) fish from Kerala, South India. Records of Zoological Survey of India 97: 61-63.

2. Ponniah AG, Gopalakrishnan A (2000) Endemic Fish diversity of the Western Ghats. NBFGR-NATP Publication, National Bureau of Fish Genetic Resources, Lucknow, India.

3. Raghavan R, Prasad G, Pereira B, Ali PHA, Sujarittanonta L (2008) 'Damse in distress'-The tale of Miss Kerala, Puntius denisonii (Day), an endemic and endangered cyprinid of the Western Ghats biodiversity hotspot (South India). Aquat Conserv 19: 67-74
4. Day F (1865) On the fishes of Cochin, on the Malabar Coast of India. Part II. Anacanthini. Proceedings of the Zoological Society of London, UK 286-318.

5. Talwar PK, Jhingran AG (1991) Inland fishes of India and Adjacent Countries Volume I, Oxford and IBH Publishing Co., New Delhi, India.

6. http://www.practicalfishkeeping.co.uk/pfk/pages/show_article.php?article id $=714$.

7. Sekharan M, Ramachandran A (2006) Market preferred indigenous ornamental fishes of Kerala. OFI Journal 52: 20-21.

8. Silas EG, Gopalakrishnan A, Ramachandran A, Anna Mercy TV, Kripan Sarkar et al. (2011) Guidelines for Green Certification of Freshwater Ornamental Fish. The Marine Products Export Development Authority, Ministry of Commerce, Govt. of India, India.

9. Lakra WS, Mohindra V, Lal KK (2007) Fish genetics and conservation research in India: status and perspectives. Fish Physiol Biochem 33: 475-487.

10. Gold JR, Richardson LR, Furman C, King TL (1993) Mitochondrial DNA differentiation and population structure in red drum (Sciaenops ocellatus) from the Gulf of Mexico and Atlantic Ocean. Marine Biol 116: 175-185.

11. Carvalho G, Pitcher T (1995) Molecular Genetics in Fisheries. (1stedn), Chapman \& Hall, London, UK.

12. Stepien CA (1999) Phylogeographical structure of the Dover sole Microstomus pacificus: the larval retention hypothesis and genetic divergence along the deep continental slope of the northeastern Pacific Ocean. Mol Ecol 8: 923-939.

13. Stamatis C, Triantafyllidis A, Moutou KA, Mamuris Z (2004) Mitochondrial DNA variation in Northeast Atlantic and Mediterranean populations of Norway lobster, Nephrops norvegicus. Mol Ecol 13: 1377-1390.

14. Piggott MP, Chao NL, Beheregaray LB (2011) Three fishes in one: cryptic species in an Amazonian floodplain forest specialist. Biol J Linn Soc Lond 102 391-403.

15. Domínguez-Domínguez O, Alda F, de León GP, García-Garitagoitia JL, Doadrio I (2008) Evolutionary history of the endangered fish Zoogoneticus quitzeoensis (Bean, 1898) (Cyprinodontiformes: Goodeidae) using a sequential approach to phylogeography based on mitochondrial and nuclear DNA data. BMC Evol Biol 8: 161.

16. Avise JC (1994) Molecular Markers, Natural History and Evolution. Springer New York, USA.

17. Moritz C, Faith DP (1998) Comparative phylogeography and the identification of genetically divergent areas for conservation. Mol Ecol 7: 419-429.

18. Wang JP, Hsu KC, Chiang TY (2000) Mitochondrial DNA phylogeography of Acrossocheilus paradoxus (Cyprinidae) in Taiwan. Mol Ecol 9: 1483-1494

19. Chen XL, Chiang TY, Lin HD, Zheng HS, Shao KT, et al. (2007) Mitochondrial DNA phylogeography of Glyptothorax fokiensis and Glyptothorax hainanensis in Asia. J Fish Biol 70: 75-93.

20. Ilves KL, Taylor EB (2009) Molecular resolution of the systematics of a problematic group of fishes (Teleostei: Osmeridae) and evidence for morphological homoplasy. Mol Phylogenet Evol 50: 163-178.

21. Benziger A, Philip S, Raghavan R, Anvar Ali PH, Sukumaran M, et al. (2011) Unraveling a 146 years old taxonomic puzzle: validation of Malabar snakehead species-status and its relevance for channid systematics and evolution. PLoS One 6: e21272.

22. Hubbs CL, Lagler KF (1947) Fishes of the Great Lakes region. Cranbrook Institute of Science, USA

23. Ward RD, Grewe PM (1994) Appraisal of molecular genetic techniques in fisheries. Rev Fish Biol Fish 4: 300-325

24. Carvalho GR, Hauser L (1994) Molecular genetics and the stock concept in fisheries. Rev Fish Biol Fish 4: 326-350.

25. Ihssen PE, Booke HE, Casselman JM, McGlade JM, Payne NR, et al. (1981) Stock identification: materials and methods. Can J Fish Aquat Sci 38: 18381855

26. Melvin GD, Dadswell MJ, McKenzie JA (1992) Usefulness of meristic and morphometric characters in discriminating populations of American shad (Alosa sapidissima) (Osteichthyes: Clupeidae) inhabiting a marine environment. Can J Fish Aqua Sci 49: 266-280.

27. Quilang JP, Basiao ZU, Pagulayan RC, Roderos RR, Barrios EB (2007) Meristic and morphometric variation in the silver perch, Leiopotherapon plumbeus (Kner, 1864), from three lakes in the Philippines. J Appl Ichthyol 23: 561-567. 
Citation: John L, Peter R, Gopalakrishnan A (2013) Population Structure of Denison's barb, Puntius denisonii (Pisces: Cyprinidae): A Species Complex Endemic to the Western Ghats of India. J Phylogen Evolution Biol 1: 106. doi:10.4172/2329-9002.1000106

28. Solem O, Berg OK (2011) Morphological differences in parr of Atlantic salmon Salmo salar from three regions in Norway. J Fish Biol 78: 1451-1469.

29. Turan C (1999) Note on the examination of the morphometric differentiation among fish populations: The Truss System. Turk Zool Derg 23: 259-263.

30. Kottelat M (1990) Indochinese nemacheilines. A revision of nemacheiline loaches (Pisces: Cypriniformes) of Thailand, Burma, Laos, Cambodia and Southern Vietnam. München.

31. Elliott NG, Haskard K, Koslow JA (1995) Morphometric analysis of orange roughy (Haplostethus atlanticus) off the continental slope of southern Australia. J Fish Biol 46: 202-220.

32. Van Valen $L$ (1978) The statistics of variation. Evolutionary Theory 4: 33-43.

33. Katselis G, Hotos G, Minos G, Vidalis K (2006) Phenotypic affinities on fry of four mediterranean grey mullet species. Turkish J Fish Aquat Sci 6: 49-55.

34. Miller SA, Dykes DD, Polesky HF (1988) A simple salting out procedure for extracting DNA from human nucleated cells. Nucleic Acids Res 16: 1215.

35. Page TJ, Sharma S, Hughes JM (2004) Deep phylogenetic structure has conservation implications for ornate rainbowfish (Melanotaeniidae: Rhadinocentrus ornatus) in Queensland, eastern Australia. Mar Freshw Res 55: 165-172.

36. Hall TA (1999) BioEdit: a user-friendly biological sequence alignment editor and analysis program for Windows 95/98/NT. Nucleic Acids Symp Ser 41: 95-98.

37. Librado P, Rozas J (2009) DnaSP v5: a software for comprehensive analysis of DNA polymorphism data. Bioinformatics 25: 1451-1452.

38. Nei M (1987) Molecular evolutionary genetics. Columbia University Press, New York, USA.

39. Tajima $F$ (1983) Evolutionary relationship of DNA sequences in finite populations. Genetics 105: 437-460

40. Excoffier L, Smouse PE, Quattro JM (1992) Analysis of molecular variance inferred from metric distances among DNA haplotypes: application to human mitochondrial DNA restriction data. Genetics 131: 479-491.

41. Wright $S$ (1951) The genetical structure of populations. Ann Eugen 15: 323354.

42. Excoffier L, Laval G, Schneider S (2007) Arlequin (version 3.0): an integrated software package for population genetics data analysis. Evol Bioinform Online 1: $47-50$

43. Tamura K, Peterson D, Peterson N, Stecher G, Nei M, et al. (2011) MEGA5 Molecular Evolutionary Genetics Analysis using maximum likelihood, evolutionary distance, and maximum parsimony methods. Mol Biol Evol.

44. Kimura M (1980) A simple method for estimating evolutionary rates of base substitutions through comparative studies of nucleotide sequences. J Mol Evol 16: $111-120$

45. Solomon S, Ramprasanth MR, Baby F, Pereira B, Tharian J, et al. (2011) Reproductive biology of Puntius denisonii, an endemic and threatened aquarium fish of the Western Ghats and its implications for conservation. J Threat Taxa 3: 2071-2077.

46. Collins RA, Armstrong KF, Meier R, Yi Y, Brown SD, et al. (2012) Barcoding and border biosecurity: identifying cyprinid fishes in the aquarium trade. PLoS One 7: e28381.

47. Bossuyt F, Meegaskumbura M, Beenaerts N, Gower DJ, Pethiyagoda R, et al. (2004) Local endemism within the Western Ghats-sri Lanka biodiversity hotspot. Science 306: 479-481.

48. Baby F, Tharian J, Philip S, Ali A, Raghavan R (2011) Checklist of the fishes of the Achankovil forests, Kerala, India with notes on the range extension of an endemic cyprinid Puntius chalakkudiensis. J Threat Taxa 3: 1936-1941.

49. Lijo John (2009) Population genetic structure of indigenous ornamental teleosts, Puntius denisonii and Puntius chalakkudiensis from the Western Ghats, India. Ph.D. Thesis, Cochin University of Science and Technology, Kerala, India.
50. Hebert PD, Cywinska A, Ball SL, deWaard JR (2003) Biological identifications through DNA barcodes. Proc Biol Sci 270: 313-321.

51. Hebert PD, Stoeckle MY, Zemlak TS, Francis CM (2004) Identification of birds through DNA Barcodes. PLoS Biol 2: e312.

52. Linares MC, Soto-Calderón ID, Lees DC, Anthony NM (2009) High mitochondrial diversity in geographically widespread butterflies of Madagascar: a test of the DNA barcoding approach. Mol Phylogenet Evol 50: 485-495.

53. Ward RD, Woodwark M, Skibinski OF (1994) A comparison of genetic diversity levels in marine, freshwater and anadromous fishes. J Fish Biol 44: 213-232.

54. McGlashan DJ, Hughes JM (2001) Low levels of genetic differentiation among populations of the freshwater fish Hypseleotris compressa (Gobiidae: Eleotridinae): implications for its biology, population connectivity and history. Heredity (Edinb) 86: 222-233

55. Carvalho GR (1993) Evolutionary aspects of fish distribution: genetic variability and adaptation. J Fish Biol 43: 53-73.

56. Mamuris Z, Apostolidis AP, Panagiotaki P, Theodorou AJ, Triantaphyllidis $C$ (1998) Morphological variation between red mullet populations in Greece. J Fish Biol 52: 107-117.

57. Hubbs CL (1922) Variations in the number of vertebrae and other meristic characters of fishes correlated with the temperature during development. Am Nat 56: 360-372.

58. Taning AV (1952) Experimental study of meristic characters in fishes. Biol Rev 27: 169-193.

59. Vasconcellos AV, Vianna P, Paiva PC, Schama R, Cava AS (2008) Genetic and morphometric differences between yellowtail snapper (Ocyurus chrysurus, Lutjanidae) populations of the tropical West Atlantic. Genetics and Molecular Biology 31: 308-316

60. Turan C, Erguden D, Turan F, Gurlek M (2004) Genetic and Morphologic Structure of Liza abu (Heckel, 1843) Populations from the Rivers Orontes, Euphrates and Tigris. Turk J Vet Anim Sci 28: 729-734.

61. Slatkin $M$ (1993) Isolation by distance in equilibrium and non-equilibrium populations. Evolution 47: 264-279.

2. Slatkin M (1987) Gene flow and the geographic structure of natural populations. Science 236: 787-792

63. Mills LS, Allendorf EW (1996) The one-migrant-per-generation rule in conservation and management. Conserv Biol 10: 1509-1518.

64. Grant WS, Bowen BW (1998) Shallow population histories in deep evolutionary lineages of marine fishes: insights from sardines and anchovies and lessons for conservation. J Hered 89: 415-426.

65. Brown WM, George M Jr, Wilson AC (1979) Rapid evolution of animal mitochondrial DNA. Proc Natl Acad Sci U S A 76: 1967-1971.

66. Alves MJ, Coelho H, Collares-Pereira MJ, Coelho MM (2001) Mitochondria DNA variation in the highly endangered cyprinid fish Anaecypris hispanica: importance for conservation. Heredity (Edinb) 87: 463-473.

67. Gopi KC (2000) Freshwater fishes of Kerala state. In: Endemic Fish Diversity of Western Ghats. NBFGR-NATP publication 1, National Bureau of Fish Genetic Resources, Lucknow, India.

68. Kurup BM, Radhakrishnan KV (2006) Freshwater fish biodiversity of Keralastatus and utilization for commercial fishing, food security and livelihood. Fishing Chimes 25: 111-122.

69. Kurup BM, Radhakrishnan KV (2006) Indigenous ornamental fish resources of Western Ghats with special reference to Kerala. In: Souvenir Publication of Ornamentals Kerala, Cochin, India.

70. Moritz C (1994) Defining 'Evolutionarily Significant Units' for conservation. Trends Ecol Evol 9: 373-375. 